Article

\title{
Numerical Analysis of Mechanical Energy Dissipation for an Axial-Flow Pump Based on Entropy Generation Theory
}

\author{
Simin Shen, Zhongdong Qian * and Bin Ji \\ State Key Laboratory of Water Resources and Hydropower Engineering Science, Wuhan University, \\ No.8 South East Lake Road, Wuhan 430072, China; emilyfluent@gmail.com (S.S.); jibin@whu.edu.cn (B.J.) \\ * Correspondence: zdqian@whu.edu.cn; Tel.: +86-027-687-74689
}

Received: 14 June 2019; Accepted: 25 October 2019; Published: 31 October 2019

\begin{abstract}
Mechanical energy dissipation is a major problem affecting hydraulic machinery especially under partial-load conditions. Owing to limitations of traditional methods in evaluating mechanical energy dissipation, entropy generation theory is introduced to study mechanical energy dissipation with varying discharge and tip clearance intuitively through numerical simulations in an axial-flow pump. Results show that the impeller and diffuser are the main domains of mechanical energy dissipation, respectively accounting for $35.32 \%-55.51 \%$ and $32.61 \%-20.42 \%$ of mechanical energy dissipation throughout the flow passage. The mechanical energy dissipation of the impeller has a strong relation with the hump characteristic and becomes increasingly important with decreasing discharge. Areas of high turbulent dissipation in the impeller are mainly concentrated near the blades' suction sides, and these regions, especially areas near the shroud, extend with decreasing discharge. When the pump enters the hump region, the distributions of turbulent dissipation near the shroud become disordered and expand towards the impeller's inlet side. Unstable flows, like flow separation and vortices, near the blades' suction sides lead to the high turbulent dissipation in the impeller and hump characteristic. Turbulent dissipation at the tip decreases from the blade leading edge to trailing edge, and regions of high dissipation distribute near the leading edge of the blade tip side. An increase in tip clearance for the same discharge mainly increases areas of high turbulent dissipation near the shroud and at the tip of the impeller, finally reducing pump performance.
\end{abstract}

Keywords: axial-flow pump; mechanical energy dissipation; entropy generation; tip clearance

\section{Introduction}

Axial-flow pumps in hydraulic projects of water supply and drainage have widespread applications, and it is thus important to study their internal flows and mechanical energy dissipation. When an axial-flow pump operates under partial-load conditions, unstable flows can result in large hydraulic losses and affect the steady operation of the pump. In studying the magnitude and distribution of mechanical energy dissipation with varying conditions, it is important to find an effective method of evaluating mechanical energy dissipation.

Traditional methods of evaluating mechanical energy dissipation, such as using the pressure drop, are limited in quantitating and locating mechanical energy dissipation. The entropy generation method overcomes these limitations, and determines the exact locations where high mechanical energy dissipation occurs and how such dissipation is distributed. Owing to its intuitive and quantitative nature, an increasing number of researchers are applying the entropy generation method in numerical simulations to investigate mechanical energy dissipation. Kock F. and Herwig H. [1-3] introduced entropy generation theory and revealed four mechanisms of entropy generation, namely heat fluxes 
due to time-averaged and fluctuating temperature fields and dissipation due to time-averaged and fluctuating velocities. Gong RZ. et al. [4] evaluated mechanical energy dissipation using entropy generation method and determined locations of dissipation in a hydro turbine. They found that more than half the mechanical energy dissipation throughout the passage occurred in the runner and nearly $25 \%$ of dissipation occurred in the guide vanes. Meanwhile most of the mechanical energy dissipation in the runner was distributed near the leading edges and trailing edges of the runner's blades. Hou et al. [5] analyzed mechanical energy dissipation in a submerged liquefied-natural-gas pump using the entropy generation method. They considered that entropy was generated by flow separation, the shock phenomenon, and vortices. Xiaojun Li et al. [6] investigated the relationship between the entropy generation field and vapor distribution. They found that the changing trend of the overall entropy generation rate of the impeller coincided with the decline of the pump head. The entropy generation rate slightly changed for cavitation at an early stage, while it greatly increased for fully developed cavitation. These high entropy generation rates occurred at the interface of the cavity, especially for the rear part of the cavity region. Pei et al. [7] studied the effects of the distance between the guide vane and impeller on the mechanical energy dissipation and distributions of this dissipation in a low-head pump applying the entropy generation method. They found that the distance affects the efficiency of the pump under positive rotation conditions and that the main mechanical energy dissipation is turbulent dissipation. Deyou Li et al. [8,9] applied the entropy generation method to analyze hysteresis characteristics of a pump-turbine model and reflected the mechanical energy dissipation directly. Their analysis revealed that the mechanical energy dissipation of the runner and guide vane resulted in hump characteristics, and dissipation was mostly in the runner. All these studies showed that the entropy generation method is effective in evaluating mechanical energy dissipation. The entropy generation method has the advantage of determining the magnitude and location of mechanical energy dissipation. Meanwhile, several researchers investigated the internal flow field in axial-flow pumps by conducting numerical simulations and physical experiments. Zhongdong Qian et al. [10] simulated complex flow in an axial-flow pump with fixed and adjustable guide vanes. Their calculation results revealed that flow separation and the attack angle play important roles in creating vortices that dissipate mechanical energy around the guide vanes. Jianjun Feng et al. [11] investigated the effects of tip clearance on pressure fluctuations and the tip clearance vortex for an axial-flow pump. Their analysis revealed that the tip clearance strongly affected the pressure fluctuations for the impeller, with no obvious effects for the diffuser. Shi et al. [12] investigated the dynamics of the tip leakage vortex and cavitation with different geometries of the tip clearance for an axial-flow pump. Desheng Zhang et al. [13] presented the dynamics and trajectory of the tip leakage vortex through numerical simulations and physical experiments for an axial-flow pump. Some other experts [14-21] investigated the complex flow in deep such as cavitating flow, tip flow and energy loss characteristics in the hydrofoil or turbo-machinery and achieved some results.

The above studies adopting the entropy generation method to investigate mechanical energy dissipation [4-9] were conducted for turbines or centrifugal pumps. Meanwhile, the main focus of our research is the exact magnitudes and distributions of mechanical energy dissipation under varying discharge and tip clearance using the entropy generation method near the hump region for an axial-flow pump. In our paper, we compare the overall entropy generation rates of varying components to illustrate the contributions of each component and how they change with varying discharge and tip clearance. We then select the components with large mechanical energy dissipation to deeply analyze changes in the distribution of dissipation with varying discharge and tip clearance and their causes. From our analysis, we draw conclusions about the quantity and distribution of mechanical energy dissipation under varying discharge and tip clearance for an axial-flow pump. Our work provides theoretical guidance for determining mechanical energy dissipation in axial-flow pumps. 


\section{Entropy Generation Theory}

According to the second law of thermodynamics, entropy generation serves as a parameter that characterizes mechanical energy dissipation due to irreversible factors during the process of energy conversions. In a real flow system of hydraulic machinery like in axial-flow pumps, mechanical energy is converted not only to pressure energy and dynamic energy but also inevitably to internal energy. Such irreversible mechanical energy dissipation can increase entropy generation. Entropy generation can therefore be applied in numerical simulations to measure such mechanical energy dissipation for axial-flow pumps.

The single-phase and incompressible flow satisfies Fourier's law of heat conduction. The transportation equation for specify entropy is written as:

$$
\rho\left(\frac{\partial s}{\partial t}+\mathrm{u} \frac{\partial s}{\partial x}+\mathrm{v} \frac{\partial s}{\partial y}+\mathrm{w} \frac{\partial s}{\partial z}\right)=-\operatorname{div}\left(\frac{\vec{q}}{T}\right)+\frac{\Phi}{T}+\frac{\Phi_{\Theta}}{T^{2}},
$$

where s is specific entropy; $\rho$ is density; $\vec{q}$ is the heat-flux density vector; the term $\frac{\Phi}{T}$ denotes the entropy generation due to dissipation; the term $\frac{\Phi_{\Theta}}{T^{2}}$ denotes entropy generation due to the temperature difference in heat transfer; $\mathrm{T}$ is temperature; and $\mathrm{u}, \mathrm{v}$, and $\mathrm{w}$ are respectively velocity components along $\mathrm{x}, \mathrm{y}$, and z directions in a Cartesian coordinate system. The terms $\frac{\Phi}{T}$ and $\frac{\Phi_{\Theta}}{T^{2}}$ are two important mechanisms for entropy generation [1]. According to Reynolds time-averaged Navier-Stokes equations, an instantaneous variable can be expressed as the sum of a time-averaged term and fluctuating term; e.g., $\mathrm{s}=\bar{s}+s$ and $\mathrm{u}=\bar{u}+\mathrm{u}^{\prime}$. Introducing these variables into Equation (1), the transportation equation becomes:

$$
\rho\left(\frac{\partial \bar{s}}{\partial t}+\bar{u} \frac{\partial \bar{s}}{\partial x}+\bar{v} \frac{\partial \bar{s}}{\partial y}+\bar{w} \frac{\partial \bar{s}}{\partial z}\right)=\overline{\operatorname{div}\left(\frac{\vec{q}}{T}\right)}-\rho\left(\frac{\partial \overline{u^{\prime} s^{\prime}}}{\partial x}+\frac{\overline{\partial v^{\prime} s^{\prime}}}{\partial y}+\frac{\overline{\partial w^{\prime} s^{\prime}}}{\partial z}\right)+\frac{\bar{\Phi}}{T}+\frac{\overline{\Phi_{\Theta}}}{T^{2}}
$$

where the term $\overline{\operatorname{div}\left(\frac{\vec{q}}{T}\right)}-\rho\left(\frac{\overline{\partial u^{\prime} s^{\prime}}}{\partial x}+\frac{\overline{\partial v^{\prime} s^{\prime}}}{\partial y}+\frac{\overline{\partial w^{\prime} s^{\prime}}}{\partial z}\right)$ represents the reversible heat transfer, this term can be neglected owing to the ignorance of heat transfer between the internal flows and outside for hydraulic machinery [14]. The term $\frac{\bar{\Phi}}{T}$ denotes the entropy generation rate induced by dissipation while the term $\frac{\Phi_{\Theta}}{T^{2}}$ denotes the energy generation rate due to heat transfer resulting from the temperature difference. Entropy generation induced by heat transfer can be neglected by regarding the internal flow of hydraulic machinery as an approximately adiabatic process. The term $\frac{\bar{\Phi}}{T}$ can thus be obtained directly for turbulent flows as:

$$
\bar{\Phi}=\dot{S}_{\bar{D}}^{\prime \prime}+\dot{S}_{D^{\prime \prime}}^{\prime \prime}
$$

which includes the mean term $\dot{S}_{\bar{D}}^{\prime \prime}$ and fluctuating term $\dot{S}_{D^{\prime}}^{\prime \prime}$. The mean term $\dot{S}_{\bar{D}}^{\prime \prime}$ is the entropy generation rate induced by time-averaged movements and represents direct dissipation. Meanwhile, the fluctuating term $\dot{S}_{\mathrm{D}^{\prime}}^{\prime \prime}$ is the entropy generation rate induced by velocity fluctuations and represents the turbulent dissipation. These two variables are calculated [4-9] as:

$$
\begin{aligned}
\dot{S}_{\bar{D}}^{\prime \prime} & =\frac{2 \mu}{T}\left[\left(\frac{\partial \bar{u}}{\partial x}\right)^{2}+\left(\frac{\partial \bar{v}}{\partial y}\right)^{2}+\left(\frac{\partial \bar{w}}{\partial z}\right)^{2}\right] \\
& +\frac{\mu}{T}\left[\left(\frac{\partial \bar{u}}{\partial y}+\frac{\partial \bar{v}}{\partial x}\right)^{2}+\left(\frac{\partial \bar{u}}{\partial z}+\frac{\partial \bar{w}}{\partial x}\right)^{2}+\left(\frac{\partial \bar{v}}{\partial z}+\frac{\partial \bar{w}}{\partial x}\right)^{2}\right],
\end{aligned}
$$




$$
\begin{aligned}
\dot{S}_{D^{\prime}}^{\prime \prime} & =\frac{2 \mu_{e f}}{T}\left[\left(\frac{\partial u^{\prime}}{\partial x}\right)^{2}+\left(\frac{\partial v^{\prime}}{\partial y}\right)^{2}+\left(\frac{\partial w^{\prime}}{\partial z}\right)^{2}\right] \\
& +\frac{\mu_{e f}}{T}\left[\left(\frac{\partial u^{\prime}}{\partial y}+\frac{\partial v^{\prime}}{\partial x}\right)^{2}+\left(\frac{\partial u^{\prime}}{\partial z}+\frac{\partial w^{\prime}}{\partial x}\right)^{2}+\left(\frac{\partial v^{\prime}}{\partial z}+\frac{\partial w^{\prime}}{\partial x}\right)^{2}\right],
\end{aligned}
$$

where $\bar{u}, \overline{\mathrm{v}}$, and $\overline{\mathrm{w}}$ are respectively mean velocity quantities along $\mathrm{x}, \mathrm{y}$, and $\mathrm{z}$ directions; $\mathrm{u}^{\prime}, \mathrm{v}^{\prime}$, and $\mathrm{w}^{\prime}$ are respectively fluctuating velocity quantities along $\mathrm{x}, \mathrm{y}$, and $\mathrm{z}$ directions; $\mu$ is the molecular viscosity; and $\mu_{\mathrm{t}}$ is the turbulent viscosity. $\mu_{\mathrm{ef}}$ is the effective viscosity and can be expressed as:

$$
\mu_{\mathrm{ef}}=\mu+\mu_{\mathrm{t}}
$$

The mean term $\dot{S}_{\bar{D}}^{\prime \prime}$ can be computed using Equation (4) through post-processing using the commercial software CFD POST. However, the fluctuating term $\dot{S}_{\mathrm{D}^{\prime}}^{\prime \prime}$ cannot be computed directly because the velocity fluctuation quantities cannot be obtained during the calculation employing the Reynolds-averaged Navier-Stokes method. According to Kock and Mathieu et al. [22,23], the turbulent entropy generation rate $\dot{S}_{D^{\prime}}^{\prime \prime}$ relates to $\varepsilon$ or $\omega$ in most turbulence models. For the $k-\varepsilon$ turbulence model, the term $\dot{\mathrm{S}}_{\mathrm{D}^{\prime}}^{\prime \prime}$ can be approximately calculated as:

$$
\dot{S}_{D^{\prime}}^{\prime \prime}=\frac{\rho \varepsilon}{T} .
$$

For the $\mathrm{k}-\omega$ turbulence model, the term $\dot{\mathrm{S}}_{\mathrm{D}^{\prime}}^{\prime \prime}$ can be computed as:

$$
\dot{S}_{D^{\prime}}^{\prime \prime}=\alpha \frac{\rho \omega k}{T},
$$

where $\alpha=0.09 ; \omega$ and $k$ are respectively the turbulent eddy frequency and turbulence kinetic energy (TKE) for the shear stress transport (SST) $k-\omega$ turbulence model. The overall entropy generation rate for a zone can be computed using the volume integration of the specific entropy generation rate:

$$
\begin{aligned}
& \dot{S}_{\bar{D}}=\int_{V} \dot{S}_{\bar{D}}^{\prime \prime} d V, \\
& \dot{S}_{D^{\prime}}=\int_{V} \dot{S}_{D^{\prime}}^{\prime \prime} d V, \\
& \dot{S}_{D}=\dot{S}_{\bar{D}}+\dot{S}_{D^{\prime}},
\end{aligned}
$$

where $\dot{S}_{\bar{D}}$ and $\dot{S}_{\mathrm{D}^{\prime}}$ are respectively the overall entropy generation rates of a zone induced by time-averaged movements and velocity fluctuations. $\dot{S}_{D}$ is the overall entropy generation rate of a zone, which is the sum of the two terms above. The terms relating to the entropy generation can therefore be obtained in numerical simulations for different domains. Meanwhile, the entropy generation method has advantages in quantitatively analyzing the mechanical energy dissipation and determining the exact locations of the dissipation.

\section{Experimental Model and Numerical Method}

\subsection{Experimental Model}

Figure 1 displays the test axial-flow pump installed in a pump-station laboratory at Wuhan University, Wuhan, China [24]. Figure 2 is a schematic of the closed-test-loop rig, which includes water tanks, gate valves, an electromagnetic flowmeter, a speed torque meter, an axial-flow pump, and a boosting pump. The electromagnetic valve and frequency converter respectively control the discharge and rotation speed of the model pump. A data acquisition system obtains parameters measured using various transducers. Parameter uncertainties in the measurements were: 
the input power $\mathrm{P} \leq 0.54 \%$, the flow rate $\mathrm{Q} \leq 0.36 \%$, the head $\mathrm{H} \leq 0.66 \%$, and the efficiency $\eta \leq 0.93 \%$. Specific.parameters of the experimental model are given in Table 1.

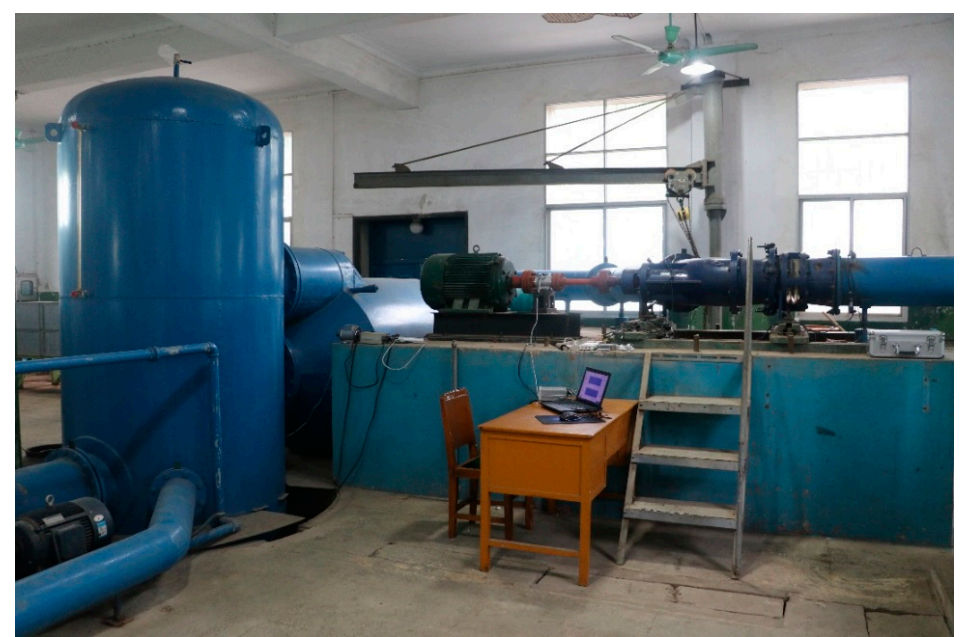

Figure 1. Axial-flow pump located at Wuhan University.

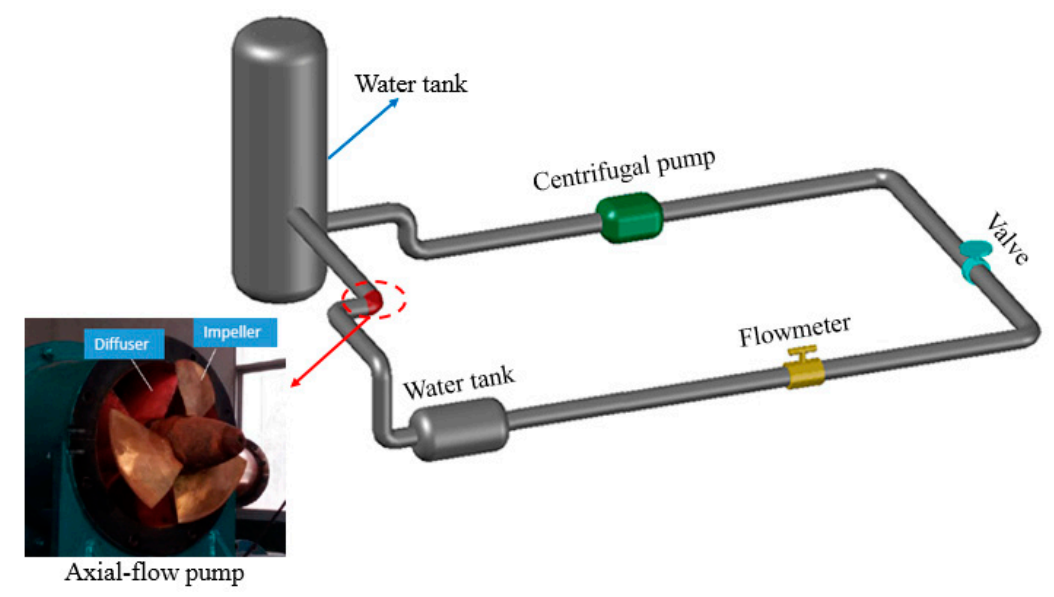

Figure 2. Schematic of the test loop.

Table 1. Main parameters of the experimental axial-flow pump

\begin{tabular}{cc}
\hline Parameters & Values \\
\hline Design flow rate $\mathrm{Q}_{\mathrm{BEP}}(\mathrm{kg} / \mathrm{s})$ & 330 \\
Design head $(\mathrm{m})$ & 3.5 \\
Diameter of Impeller $(\mathrm{m})$ & 0.3 \\
Blade number of Impeller & 3 \\
Blade number of Diffuser & 5 \\
Specific speed $\mathrm{n}_{\mathrm{q}}$ & 340 \\
Rotational speed $\mathrm{n}(\mathrm{rpm})$ & 1450 \\
Design tip clearance of Impeller $(\mathrm{mm})$ & 0.15 \\
\hline
\end{tabular}

\subsection{Computational Domain and Grid Generation}

The computational domain is the entire flow passage, including the inlet pipe, guide cone, impeller, diffuser, exit cone, $60^{\circ}$ bend, and outlet pipe [25-27], as shown in Figure 3. To ensure the flow field from the guide cone to the $60^{\circ}$ bend is independent of boundary conditions, we set the lengths of the inlet and outlet pipe as 10 times the pipe diameter. The pipe diameter was $350 \mathrm{~mm}$. Only the impeller rotated while other parts were stationary. In the impeller domain, the entire hub rotated while the 
shroud was stationary. The sliding interfaces were set at split planes of the domain of the impeller, as shown in Figure 3.

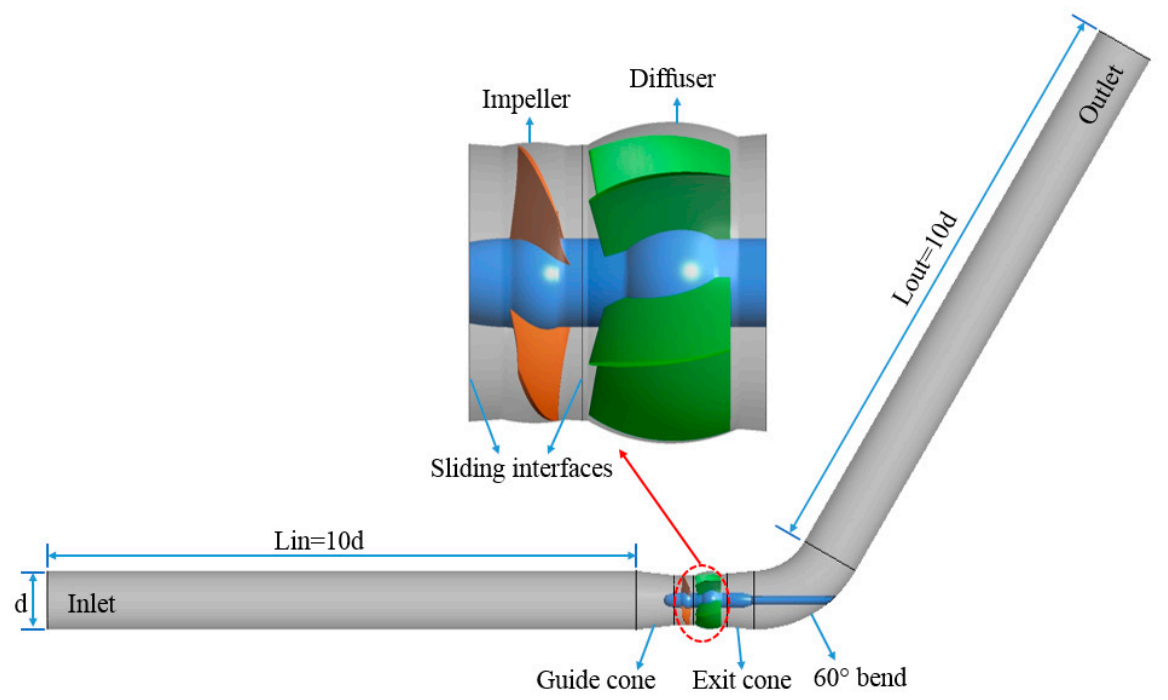

Figure 3. Three-dimensional view of the computational zone.

To achieve better convergence, structured hexahedral grids were generated for all components in the computational zone. All grids except those of the impeller were created using the software ANSYS ICEM. Owing to the high distortion of the impeller's blades and small radial tip clearance, the grids of the impeller were generated using the software ANSYS TURBOGRID; in particular, O-Grid meshes were generated around blades. Additionally, 80 layers of grids were set in the tip clearance width of $1.2 \mathrm{~mm}$. For different tip clearance widths of $0.15,0.45$, and $0.9 \mathrm{~mm}$ in this study, a uniform tip gap was applied as for the tip clearance of $1.2 \mathrm{~mm}$ in simulations. To generalize the results for the tip clearance, we should specify the tip clearance according to a fraction of the span. Therefore, we respectively converted the tip clearance widths of $0.15,0.45,0.9$, and $1.2 \mathrm{~mm}$ to $0.1 \%, 0.3 \%, 0.6 \%$, and $0.8 \%$ span in the following. Figure 4 presents the produced grids of the main components of the pump.

Four sets of the mesh system for the entire computational domain at the same discharge $(\mathrm{Q}=230.748 \mathrm{~kg} / \mathrm{s})$ are generated as listed in Table 2, and a total of 4158353 cells were found to be sufficient for simulations through validation of grid independence. Owing to the flow separation and vortices that can form in the pump passage, the SST $k-\omega$ turbulence model was applied in the simulations for the pump. The wall y+ values of cells of components, such as those around the impeller's blades, were mostly below 100, which is reasonable for numerical simulations using the SST $\mathrm{k}-\omega$ turbulence model.

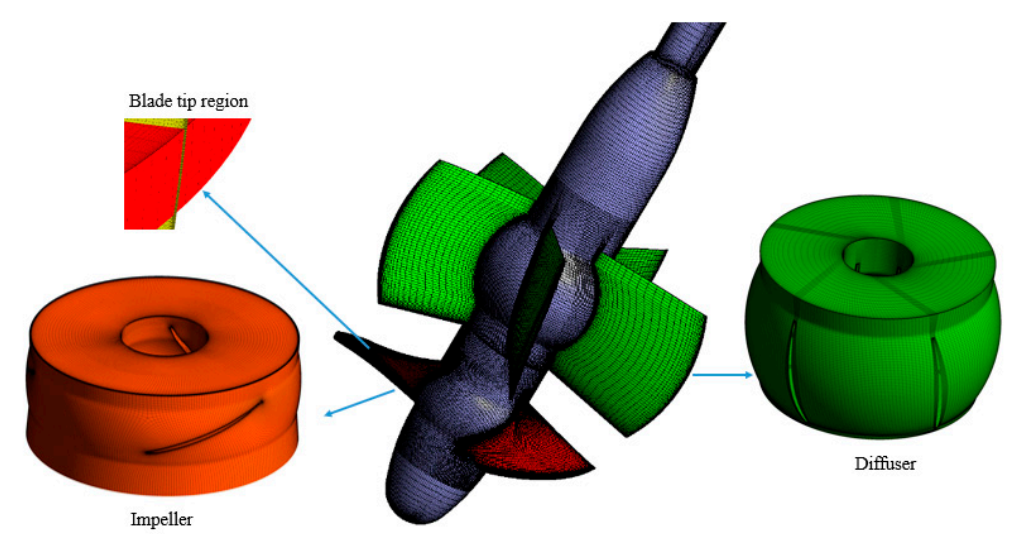

Figure 4. Grids of the main components of the axial-flow pump. 
Table 2. Verification of grid independence.

\begin{tabular}{ccccc}
\hline Total Mesh Elements & Impeller Mesh Elements & Convergence & Simulation Head (m) & Relative Error with Experiment \\
\hline 2051949 & 970191 & $10^{-5}$ & 4.985 & $9.425 \%$ \\
3086840 & 1365390 & $10^{-5}$ & 5.271 & $4.511 \%$ \\
4158353 & 2078340 & $10^{-5}$ & 5.350 & $2.787 \%$ \\
5164385 & 2748135 & $10^{-5}$ & 5.347 & $3.134 \%$ \\
\hline
\end{tabular}

\subsection{Numerical Settings}

The present study employs the commercial software ANSYS FLUENT to conduct simulations and software CFD POST as a post-processing tool for the axial-flow pump. To improve the accuracy of simulation results, we here conducted transient calculations with the SST k- $\omega$ turbulence model. We applied the finite volume method to discretize the governing equations accompanying the (second-order upwind) SIMPLEC algorithm in the simulation. The mass flow rate was set as the inlet boundary and the pressure was specified for the outlet boundary. A no-slip wall was set as the wall boundary. For transient computations, the time step was $0.0003448276 \mathrm{~s}$, which corresponded to $1 / 120$ of the rotating cycle at the designed rotating speed. For each time step, the maximum number of iterations was set at 20. The sliding interfaces at split planes of the impeller domain were specified as the 'transient rotor-stator'. The results of steady-state calculations were taken as the initial values for unsteady calculations. The total unsteady calculation was performed for 14 rotating cycles [16]. Different average values of the results of the last two, four and six periods of transient calculation were selected for analysis; the errors among these average values were less than $5 \%$. Thus, the last four time periods of the calculation were sufficient for analysis and we took these as our time sample. All results that we used for analysis in the following were based on the average result for this time sample.

\section{Results and Discussion}

\subsection{Verification of Numerical Simulations}

Figure 5 compares the pump performance between experiments and numerical simulations. The non-dimensional parameters used in the figure were calculated as:

$$
\begin{aligned}
& \text { flow coefficient : } \varphi=\frac{Q}{\left(\frac{\Omega}{2 \pi}\right) D^{3}} . \\
& \text { head coef ficient : } \psi=\frac{g H}{\left(\frac{\Omega}{2 \pi}\right)^{2} D^{2}} .
\end{aligned}
$$

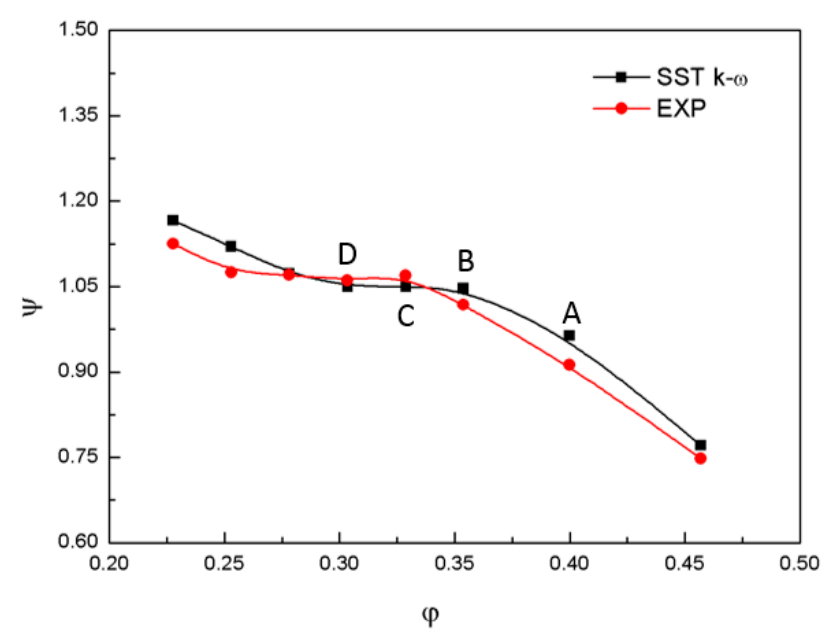

Figure 5. Comparison of simulation results and experimental data. 
Figure 5 shows that the simulation results for a tip clearance of $0.1 \%$ span had a variation trend similar to that of the experimental data, with the maximum relative error being around $5 \%$. Thus, we considered that the simulation results were reliable for current estimates. To study the relationship between the entropy generation and pump performance near the hump region, we selected the four operation points $\mathrm{A}(\varphi=0.4), \mathrm{B}(\varphi=0.354), \mathrm{C}(\varphi=0.329)$, and $\mathrm{D}(\varphi=0.303)$ displayed in Figure 5 for deep analysis in the following. Additionally, point $\mathrm{B}$ near the start of the hump region was later selected to investigate the effects of the four tip clearance widths of $0.1 \%, 0.3 \%, 0.6 \%$, and $0.8 \%$ span on mechanical energy dissipation for the axial-flow pump.

\subsection{Overall Entropy Generation Rates of Different Components for Varying Discharge}

To generalize the results of the entropy generation, we used variables $S_{D M}, S_{D E}$, and $S_{D Z}$ to reflect the turbulent entropy generation rate, the overall entropy generation rate for a domain, and the overall turbulent entropy generation rate for a domain respectively. These variables analyzed in the following were calculated as:

$$
\begin{gathered}
S_{D M}=\frac{\dot{\mathrm{S}}_{\mathrm{D}^{\prime}}^{\prime \prime}}{\rho U_{\text {tip }}^{2} T_{r e f}}, \\
S_{D E}=\frac{\dot{\mathrm{S}}_{\mathrm{D}}}{\rho U_{\text {tip }}^{2} V_{\mathrm{Z}} T_{r e f}}, \\
S_{D Z}=\frac{\dot{\mathrm{S}}_{\mathrm{D}^{\prime}}}{\rho U_{\text {tip }}^{2} V_{Z} T_{r e f}}, \\
U_{\text {tip }}=\Omega * \mathrm{Z}_{\mathrm{R}} / 2=22.80(\mathrm{~m} / \mathrm{s}),
\end{gathered}
$$

where $\rho$ is water density; $U_{\text {tip }}$ is the blade tip speed and can be calculated by Equation (17); $V_{Z}$ is the volume of the entire passage; $T_{r e f}$ is the inlet temperature $(298 \mathrm{~K}) ; \dot{\mathrm{S}}_{\mathrm{D}^{\prime}}^{\prime \prime}$ is the entropy generation rate caused by velocity fluctuation; $\dot{S}_{\mathrm{D}}$ is the overall entropy generation rate for a domain; and $\dot{S}_{\mathrm{D}^{\prime}}$ is the overall entropy generation rate caused by velocity fluctuations for a domain.

Figure 6 presents the $S_{D E}$ (reflecting overall entropy generation rate for a domain) of different components for the pump with four discharges and a tip clearance of $0.1 \%$ span. It is seen that the curves of the overall entropy generation rates for the whole passage and the impeller both increase greatly with a similar trend as the discharge reduces, and the impeller is the component with the largest mechanical energy dissipation. Meanwhile, the overall entropy generation rate increased from the guide cone to the inlet pipe, outlet pipe, $60^{\circ}$ bend, exit cone, and diffuser. As the discharge decreased, the overall entropy generation rates of the diffuser, exit cone, and $60^{\circ}$ bend increased slowly while those of the guide cone, outlet pipe, and inlet pipe remained stable and low. Additionally, as the discharge reduces from $\varphi=0.354$ to $\varphi=0.303$, the growth rates of overall entropy generation for the whole passage and the impeller were faster than those for the change from $\varphi=0.4$ to $\varphi=0.354$. Additionally, we see from Figure 5 that the start of the hump region of the pump was around $\varphi=0.354$. This means the mechanical energy dissipation of the whole passage and impeller increased more rapidly after the pump entered its hump region. This demonstrated that the mechanical energy dissipation in the impeller had a strong relation with the hump characteristic.

Figure 7 shows changes in $E_{R}$ for different discharges, where $E_{R}$ is the percentage contribution of the overall entropy generation rate $\dot{S}_{D}$ of a particular component to the overall entropy generation rate of the whole passage. The figure shows that $\mathrm{E}_{\mathrm{R}}$ was less than $7 \%$ for the guide cone, outlet pipe, and inlet pipe and largely within the range of $8 \%-13 \%$ for the exit cone and $60^{\circ}$ bend. The overall entropy generation rates of the guide cone, outlet pipe, and inlet pipe all accounted for small proportions of the total flow passage and changed little with the discharge. They could therefore be ignored when analyzing the mechanical energy dissipation. Meanwhile, the percentage contributions for the impeller and diffuser were relatively large, with the components respectively accounting for $35.32 \%-55.51 \%$ 
and $32.61 \%-20.42 \%$ of the overall entropy generation rate of the whole passage for the four discharges. Additionally, $\mathrm{E}_{\mathrm{R}}$ for the impeller increased while $\mathrm{E}_{\mathrm{R}}$ for the diffuser declined with a decreasing flow rate. The analysis indicates that the impeller and diffuser were the main locations of mechanical energy dissipation for the pump, and the dissipation in the impeller became more important with a reducing flow rate. We should therefore pay more attention to mechanical energy dissipation of the impeller and diffuser for the axial-flow pump.

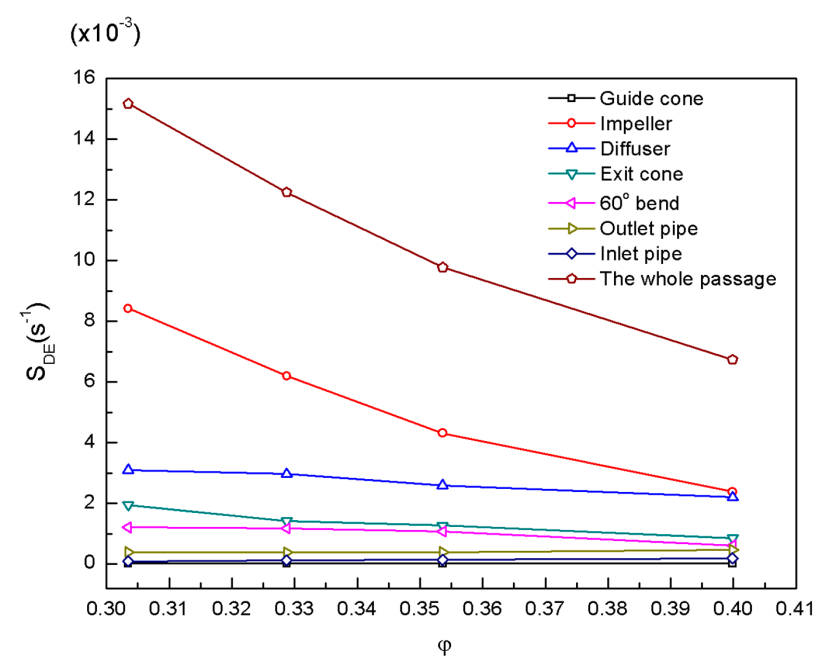

Figure 6. The $S_{D E}$ (reflecting the overall entropy generation rate for a domain) of different components for four discharges.

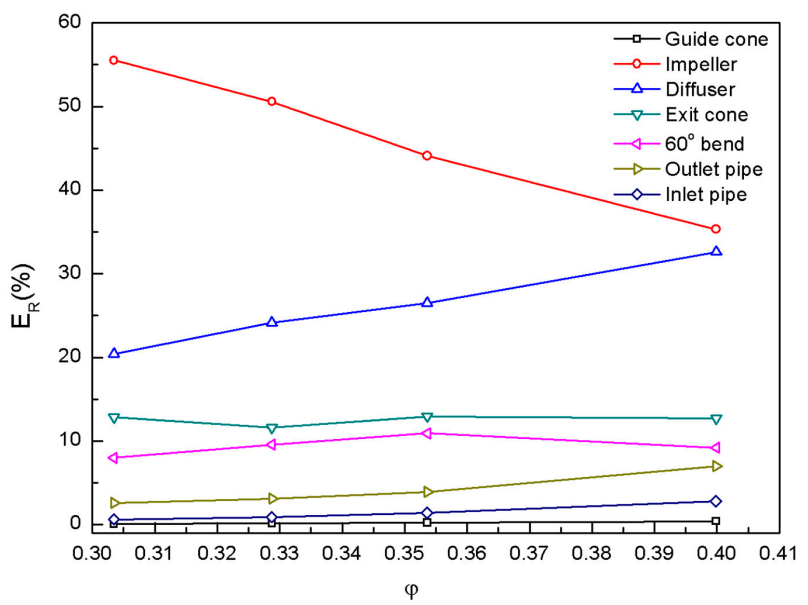

Figure 7. Percentage contributions of $\dot{S}_{D}$ of different components to the overall entropy generation rate of the whole flow passage for four discharges.

Figure 8 presents changes in $\mathrm{M}$ with varying discharge, where $\mathrm{M}$ is the percentage contribution of the overall turbulent entropy generation rate $\dot{S}_{\mathrm{D}^{\prime}}$ of a component to the overall entropy generation rate $\dot{S}_{D}$ for the same component. The figure shows that $M$ was large for all domains, ranging $94 \%-100 \%$, under different conditions. Therefore, most of the mechanical energy dissipation in the pump was induced by turbulent dissipation, and turbulent entropy generation rate should be mainly analyzed in the following.

Figure 9 displays the $S_{D Z}$ (reflecting the overall turbulent entropy generation rates for a domain) of different components for four discharges and the same tip clearance of $0.1 \%$ span. The value $S_{D Z}$ had a trend similar to that of the $S_{D E}$ in Figure 6. As the flow rate decreased, the turbulent entropy generation rates of the diffuser, exit cone, and $60^{\circ}$ bend increased slowly, while the turbulent entropy 
generation rate of the impeller increased greatly. Therefore, the discharge had greater effects on the turbulent dissipation in the impeller when the axial-flow pump entered the hump region.

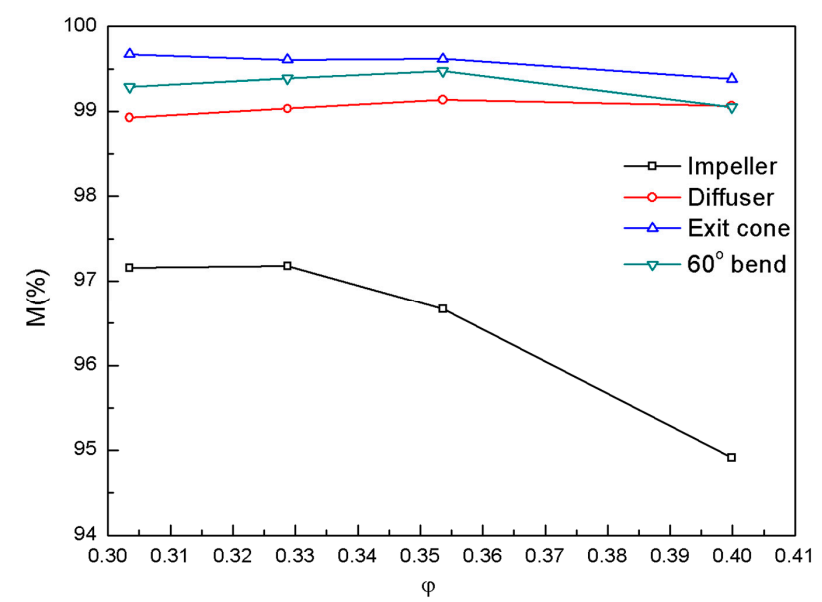

Figure 8. Percentage contributions of the overall turbulent entropy generation rate $\dot{S}_{D^{\prime}}$ of a component to the overall entropy generation rate for the same component with four discharges.

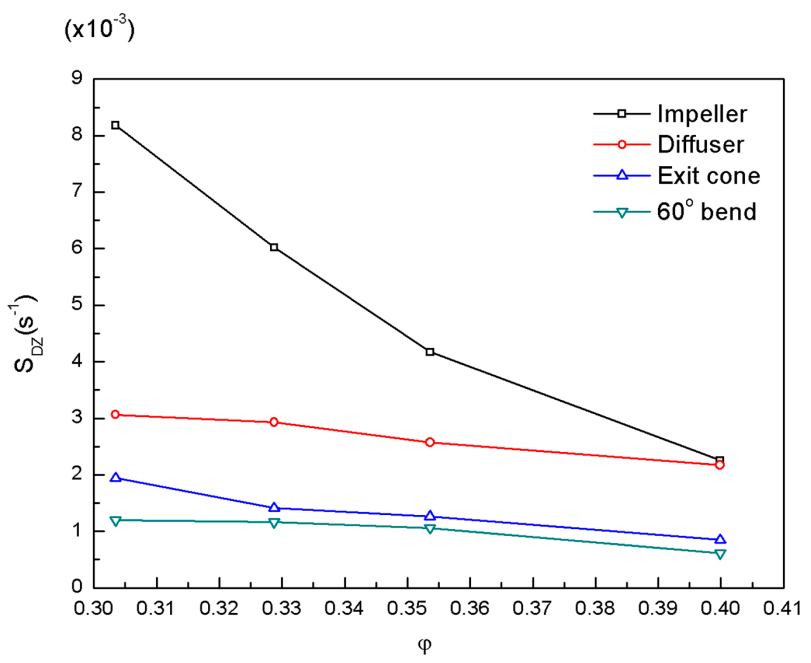

Figure 9. The $S_{D Z}$ (reflecting the overall turbulent entropy generation rate for a domain) of different components for four discharges.

\subsection{Overall Entropy Generation Rates of Different Components for Varying Tip Clearance}

There is an inevitable tip clearance between the impeller's blade and shroud when an axial-flow pump operates. To study effects of the tip clearance width on overall entropy generation rates of different components, we selected point B $(\varphi=0.354)$ near the start of the pump's hump region with four tip clearance widths to conduct the analysis work. Figure 10 shows the pump performance for the four tip clearances under the same operating condition. It is seen that the pump performance decreased with the tip clearance increasing. The head of the pump reduced quickly as the tip clearance increased from $0.3 \%$ span to $0.8 \%$ span, indicating that a tip clearance larger than $0.3 \%$ span strongly affected the pump performance.

The above analysis revealed that more than $94 \%$ of the mechanical energy dissipation was induced by turbulent dissipation. We therefore computed the overall turbulent entropy generation rates for different domains for the axial-flow pump. Figure 11 presents the $S_{D Z}$ (reflecting the overall turbulent entropy generation rate for a domain) of different domains with varying tip clearance at $\varphi=0.354$. Figure 11 clearly shows that the turbulent entropy generation rate for the impeller increased while the turbulent entropy generation rates for the diffuser and exit cone slowly decreased with the tip clearance 
increasing. Additionally, the turbulent entropy generation rate for the $60^{\circ}$ bend fluctuated within a small range. Meanwhile, the overall turbulent entropy generation rate increased from the $60^{\circ}$ bend to the exit cone, diffuser, and impeller. The impeller therefore remained the main location of mechanical energy dissipation for different tip clearances. When the tip clearance grew from $0.3 \%$ span to $0.8 \%$ span, the turbulent dissipation in the impeller increased rapidly, indicating that the turbulent entropy generation was related to the decline in pump performance as shown in Figure 10. A tip clearance larger than $0.3 \%$ span thus affected the turbulent dissipation of the impeller greater and finally affected the pump performance.

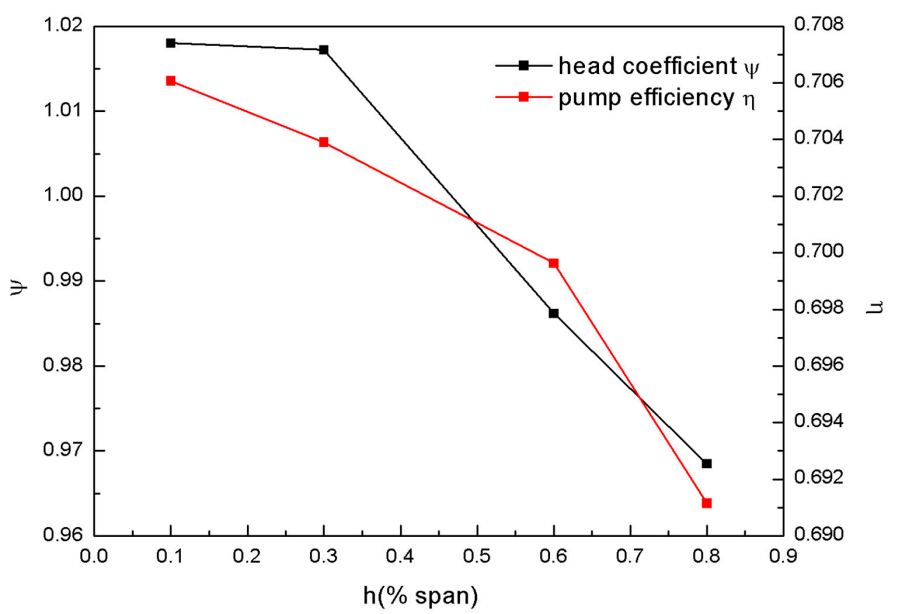

Figure 10. Axial-flow pump performance with varying tip clearance at $\varphi=0.354$.

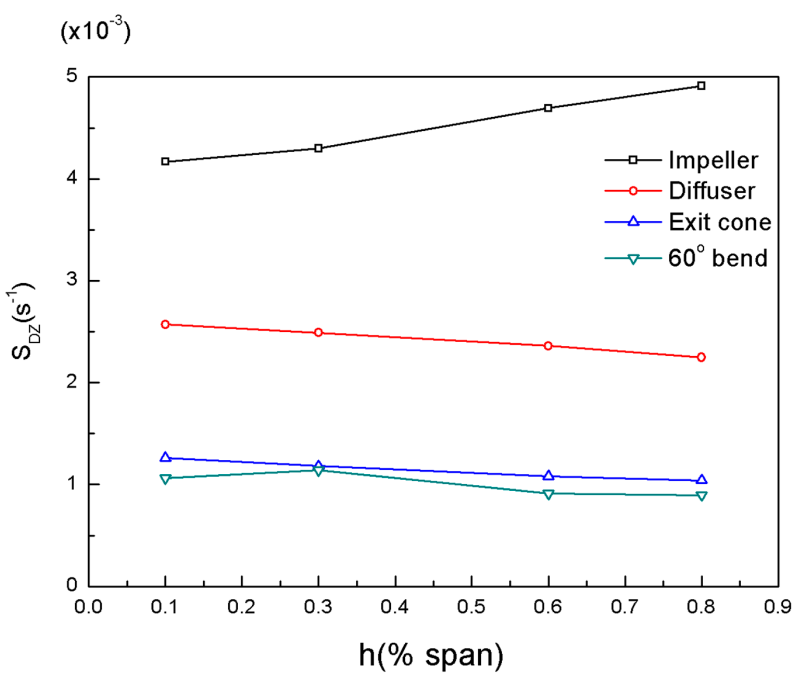

Figure 11. The $S_{D Z}$ (reflecting the overall turbulent entropy generation rate for a domain) of different domains with varying tip clearance at $\varphi=0.354$.

\subsection{Detailed Distributions of the Turbulent Entropy Generation Rate with Varying Discharge}

The above analysis showed that the impeller and diffuser were the main locations of mechanical energy dissipation and that most of the power was lost through turbulent dissipation. The turbulent entropy generation rates for the impeller and diffuser were thus selected for analysis here. To determine exact locations of turbulent dissipation, contours of the turbulent generation rate for the impeller were drawn along different spanwise surfaces under four discharges. The spanwise surface was set from 0 to 1, corresponding from the impeller's hub to the shroud; SP0.2 was near the hub and SP0.98 was near the shroud. Figure 12 presents the distributions of $S_{D M}$ of the impeller along different spans (SP0.2, SP0.5, SP0.8, and SP0.98) for four discharges. In Figure 12a, areas of high $\mathrm{S}_{\mathrm{DM}}$ were 
mainly near the leading edges of blades' suction surfaces, and the span SP0.98 near the shroud having the largest areas of turbulent dissipation. Meanwhile, there were areas of relatively high turbulent dissipation in the blade wake region and in the flow passage of the impeller as shown in Figure 12a. When the discharge reduced to operation point $B$ as shown in Figure 12b, distributions of the turbulent generation rate along the spans were similar to those in Figure 12a, while areas of high $\mathrm{S}_{\mathrm{DM}}$ were obviously larger than those in Figure 12a for each corresponding span and especially for span SP0.98. With the discharge further decreasing to point $C$ in Figure 12c, the areas of high $S_{D M}$ further grew for each corresponding span relative to the areas in Figure 12b. For the span SP0.98 shown in Figure 12c, areas of high $\mathrm{S}_{\mathrm{DM}}$ became disordered and expanded towards the inlet of the impeller. As for the lowest discharge of operation point $\mathrm{D}$ displayed in Figure 12d, areas of high $\mathrm{S}_{\mathrm{DM}}$ extended further for span SP0.98. The distributions of high turbulent dissipation on surfaces SP0.8 and SP0.98 displayed in Figure $12 \mathrm{~d}$ became more disordered and extended more towards the inlet of the impeller relative to the distributions of Figure 12c. The above analysis reveals that turbulent dissipation of the impeller was mainly concentrated near blades' suction sides and that the span SP0.98 near the shroud had the largest areas of turbulent dissipation for four operation points. Areas of high turbulent dissipation of the impeller extend with a decreasing flow rate, especially at the span near the shroud. Additionally, when the pump entered the hump region of points $C$ and $D$, the distributions of turbulent dissipation near the shroud became disordered and expanded towards the impeller's inlet side.

To explain the distributions of turbulent dissipation in the impeller, flow patterns around the blade suction side as presented by the iso-surface of TKE are investigated for four discharges. Figure 13 displays the iso-surface of TKE (for a value of 0.017) and iso-lines of non-dimensional TKE around the blade for varying discharge and a tip clearance of $0.1 \%$ span. It is seen that regions of high TKE (red areas in Figure 13) concentrated near the blade's suction surface and the location of the tip leakage vortex near the shroud had high TKE under four discharges. Additionally, areas of high TKE, especially those near the shroud, grew with a decreasing flow rate. It is thus clear that the distributions of areas having a high TKE in Figure 13 correspond with the distributions of areas having a high turbulent entropy generation rate in Figure 12, indicating that the regions of high turbulent dissipation strongly correlated with areas of high TKE. To deeply study the cause of high TKE near the shroud, we drew the non-dimensional velocity vector at span SP0.98 around one blade of the impeller with varying discharge under the same tip clearance of $0.1 \%$ span displayed in Figure 14 for analysis. As seen from Figure 14a, the flow field was relatively smooth because the difference between the flow angle and blade setting angle was small. The high turbulent dissipation near the leading edge of the blade suction side was mainly due to the flow attack and separation. The flow separation was caused by the pressure difference between the blade suction side and blade pressure side. In Figure 14b, we see an attack angle larger than that displayed in Figure 14a. This is because the difference between the flow angle and blade setting angle increased as the flow rate reduced. The flow separation increased because of greater pressure differences between the pressure and suction sides of blades, which generated higher turbulent dissipation near the shroud. Additionally, there was a low-velocity area near the blade suction side and wake area because of tip leakage vortex and wake flows. When the discharge further decreased to point $C$, the flow field near the shroud displayed in Figure 14c became disordered and there was a backflow area. This is because the flow attack angle displayed in Figure 14c was larger than that displayed in Figure $14 \mathrm{~b}$ and produced greater flow separation and vortices. Thus, the high level of turbulent dissipation near the shroud was mainly induced by the flow separation and vortices of leakage flows, backflows and wake flows. As for point $D$, the flow field near the shroud displayed in Figure 14d is was more unstable than that displayed in Figure 14c because of the larger flow attack angle. Meanwhile, there were two backflow areas owing to greater flow separation and vortices than those displayed in Figure 14c. Additionally, the areas of vortices and flow separation had high TKE. Thus, unstable flows like flow separation and vortices form near the blade suction side, high TKE accumulated in these areas, and there was finally large turbulent dissipation there. These unstable flows like flow separation and vortices of backflows near the blade suction side led to 
the hump characteristic. This explains why most turbulent dissipation concentrated near the blades' suction sides and why large areas of turbulent dissipation grew especially near the shroud with a reducing flow rate.

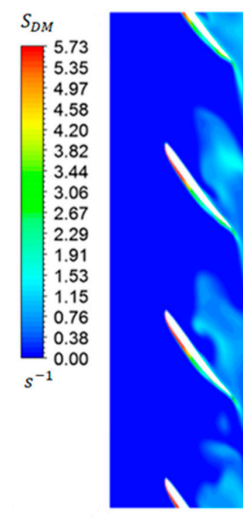

SP0.2

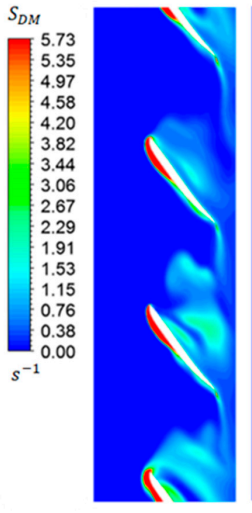

SP0.2

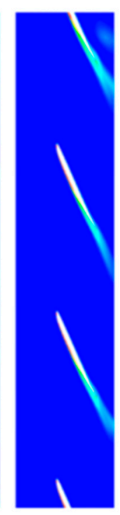

SP0.5

(a)

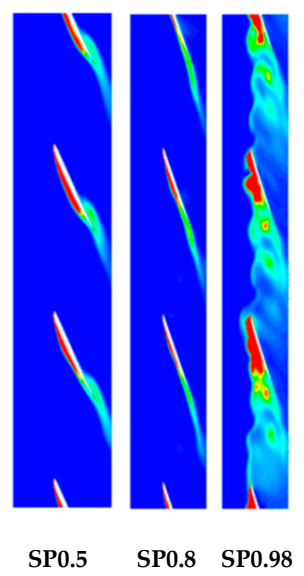

(c)

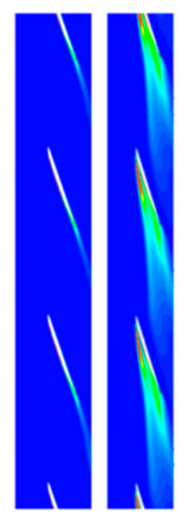

SP0.8 SP0.98
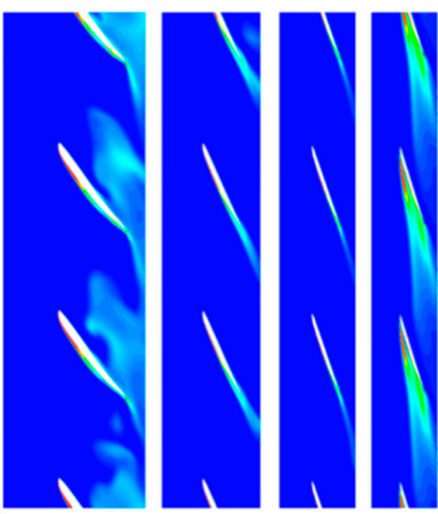

SP0.2

$\begin{array}{lll}\text { SP0.5 } & \text { SP0.8 } & \text { SP0.98 }\end{array}$

(b)

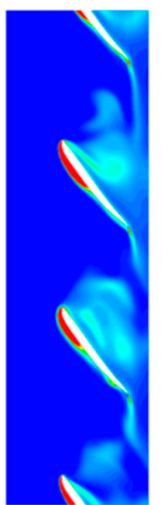

SP0.2

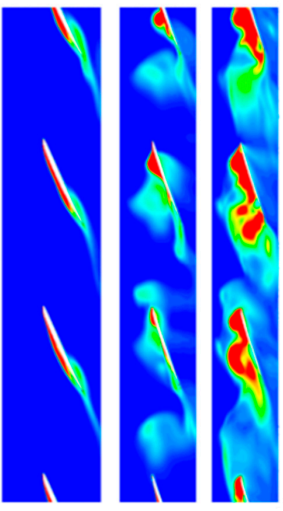

$\begin{array}{lll}\text { SP0.5 } & \text { SP0.8 } & \text { SP0.98 }\end{array}$

Figure 12. Distributions of $S_{D M}$ (reflecting the turbulent entropy generation rate) along different spans for the impeller with varying discharge $((\mathbf{a}) \varphi=0.4 ;(\mathbf{b}) \varphi=0.354 ;(\mathbf{c}) \varphi=0.329$; and (d) $\varphi=0.303)$.

As for the overall turbulent entropy generation rate of the diffuser, the turbulent dissipation increased slowly as the flow rate decreased as displayed in Figure 9. To localize the large turbulent dissipation under varying discharge, we drew contours of $S_{D M}$ (reflecting the turbulent generation rate) for the diffuser along different spanwise surfaces for analysis. Figure 15 presents the distribution of $\mathrm{S}_{\mathrm{DM}}$ for the diffuser along different spans (SP0.2, SP0.5, SP0.8, and SP0.98) with four discharges. The figure shows that regions of high $S_{D M}$ located near the leading edges of guide vanes and in the passage of the diffuser, and the span SP0.98 near the wall had the largest turbulent dissipation for the four discharges. With the flow rate decreasing, the distribution of high turbulent dissipation spread moderately and became more uniform especially for the span SP0.98 near the wall. The flow attacking owing to a difference between the flow angle and the setting angle of guide vanes was responsible for the high turbulent dissipation at the leading edges of the guide vanes in the diffuser. Additionally, the vortices in the passage lead to areas of relatively high turbulent dissipation in the diffuser. All in all, most high turbulent dissipation was near the leading edges of guide vanes and in the diffuser passage, and the distribution of high turbulent dissipation expanded to be more uniform as the flow rate declined. 


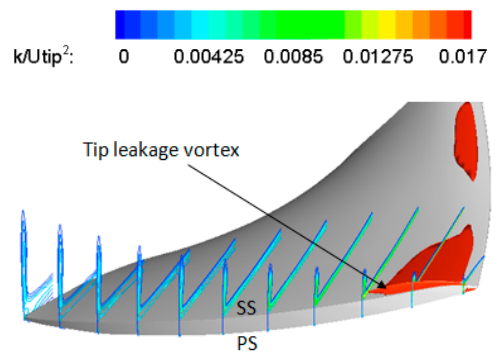

(a)

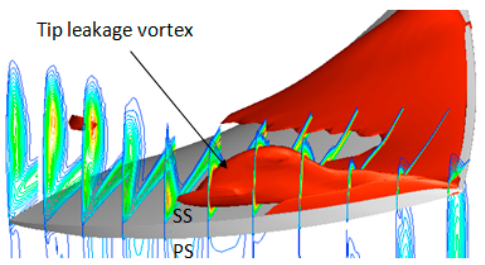

(c)

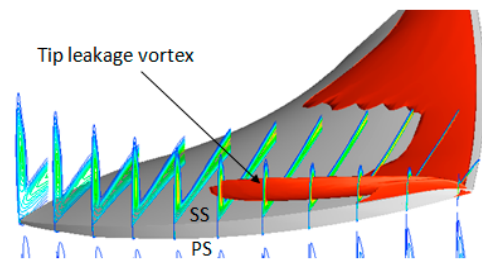

(b)

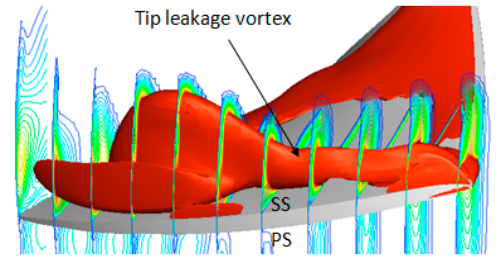

(d)

Figure 13. Flow patterns for varying discharges around the blade. Isosurface of non-dimensional turbulence kinetic energy (TKE) for a value of 0.017 and magnified isolines of non-dimensional TKE $((\mathbf{a}) \varphi=0.4 ;(\mathbf{b}) \varphi=0.354 ;$ (c) $\varphi=0.329$; and (d) $\varphi=0.303)$.

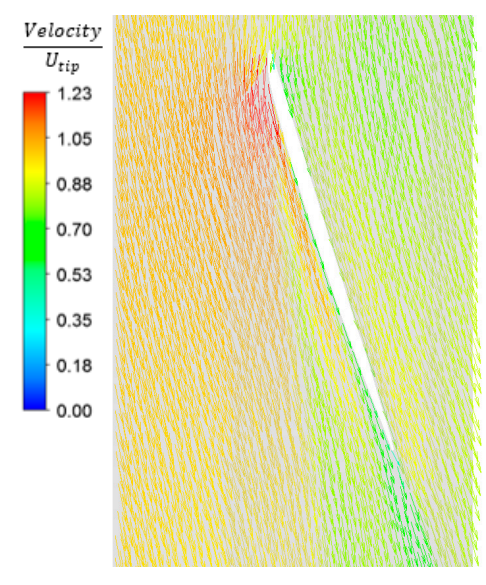

(a)

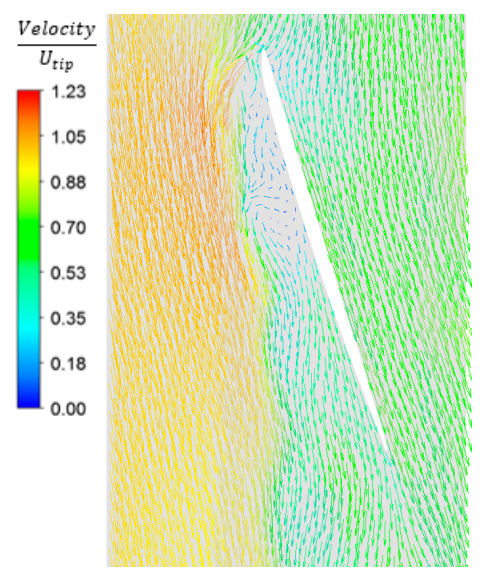

(c)

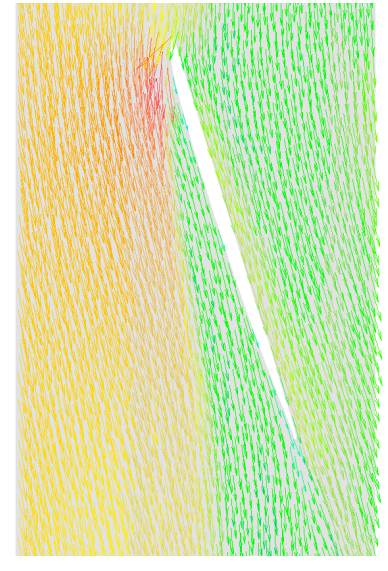

(b)

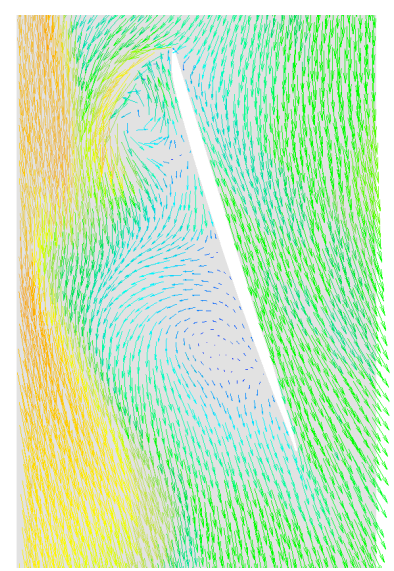

(d)

Figure 14. Non-dimensional velocity vector at the span SP0.98 for the impeller with varying discharge under a tip clearance of $0.1 \% \operatorname{span}((\mathbf{a}) \varphi=0.4 ;(\mathbf{b}) \varphi=0.354$; (c) $\varphi=0.329$; and (d) $\varphi=0.303)$. 


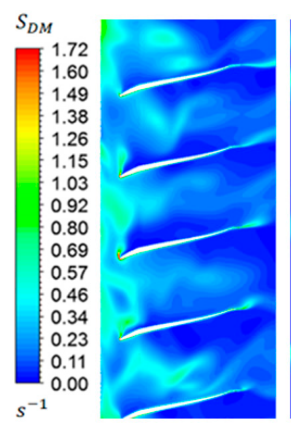

SP0.2

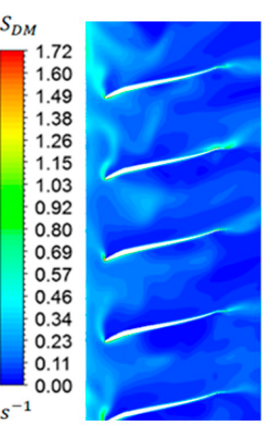

SP0.2

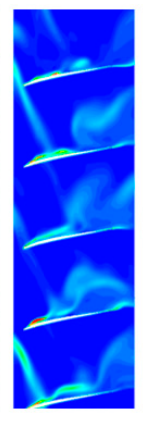

SP0.5

(a)

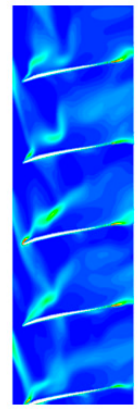

SP0.5

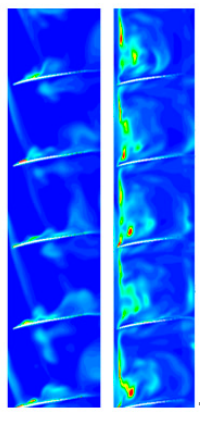

SP0.8 SP0.98

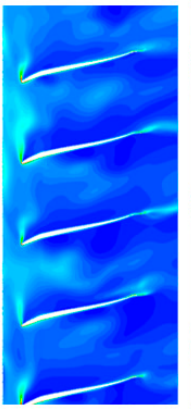

SP0.2

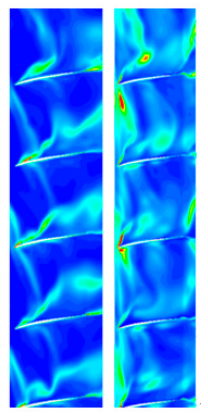

SP0.8 SP0.98

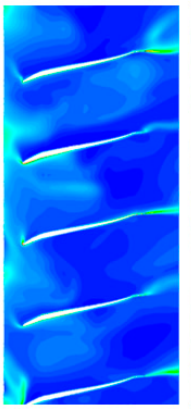

SP0.2

(c)

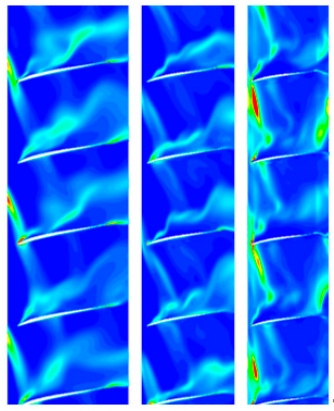

$\begin{array}{lll}\text { SP0.5 } & \text { SP0.8 } & \text { SP0.98 }\end{array}$

(b)

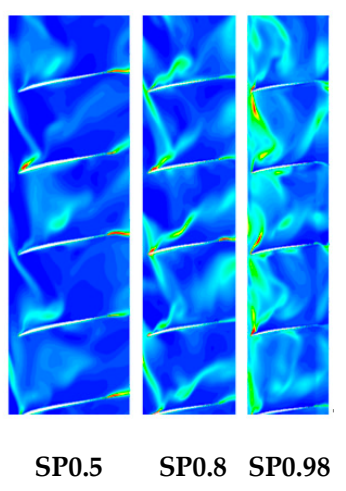

(d)

Figure 15. Distributions of $S_{D M}$ (reflecting the turbulent entropy generation rate) on different spanwise surfaces of the diffuser with varying discharge $((\mathbf{a}) \varphi=0.4$; (b) $\varphi=0.354$; (c) $\varphi=0.329$; and (d) $\varphi=0.303$ ).

\subsection{Detailed Distributions of the Turbulent Entropy Generation Rate with Varying Tip Clearances}

Figures 10 and 11 show that the overall turbulent entropy generation rate of the impeller increases with the tip clearance increasing from $0.1 \%$ span to $0.8 \%$ span and this was strongly related with a decline in pump performance. The turbulent entropy generation rate of the impeller was therefore chosen for analyzing effects of the tip clearance width on mechanical energy dissipation for the same discharge $(\varphi=0.354)$. Similar to the analysis above, we drew contours of $S_{D M}$ (reflecting the turbulent generation rate) along different spans of the impeller to determine the magnitude and locations of turbulent dissipation. Figure 16 shows the distributions of $\mathrm{S}_{\mathrm{DM}}$ of the impeller along different spans (SP0.2, SP0.5, SP0.8, and SP0.98) for four tip clearance widths at operation point B. Figure 16a is the same as Figure $12 \mathrm{~b}$, showing that regions having a high turbulent entropy generation rate were mainly distributed near blades' suction sides. When the tip clearance grew from $0.1 \%$ span to $0.8 \%$ span as shown in Figure 16, regions of high turbulent dissipation on surface SP0.98 increased and expanded along blades' suction sides in the passage of the impeller, and these regions of high turbulent dissipation even approached the pressure sides of neighboring blades in the case of the largest tip clearance of $0.8 \%$ span. As found in our previous research on tip flows [25], the tip clearance width mainly affected the flow structure near the shroud and more vortices were generated near the shroud with the tip clearance increasing. Figure 16 shows that areas of high turbulent dissipation grew obviously for span SP0.98 with the tip clearance increasing at the same discharge $(\varphi=0.354)$. Therefore, an increase in tip clearance mainly increased turbulent dissipation near the shroud of the impeller and finally reduced the pump performance. 


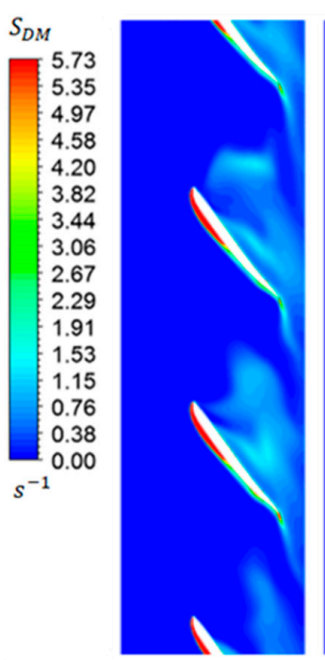

SP0.2

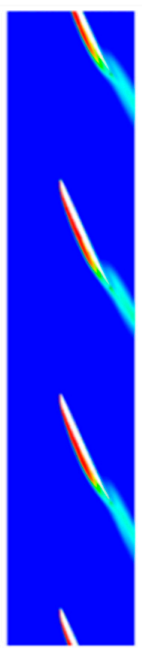

SP0.5

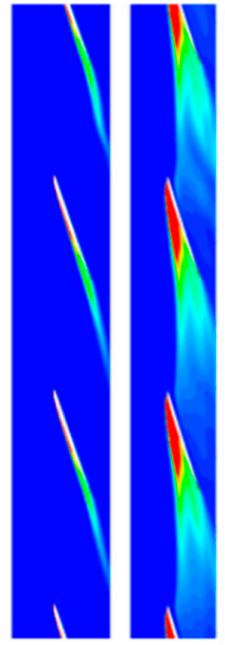

SP0.8 SP0.98

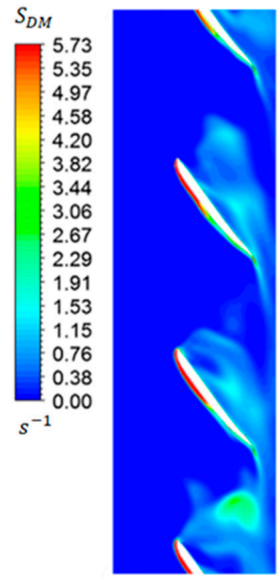

SP0.2 (a)

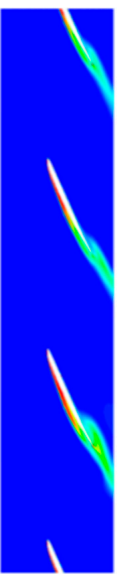

SP0.5

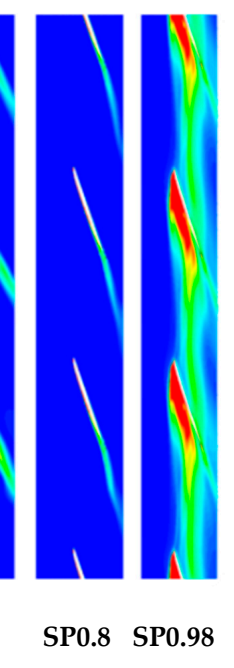

(c)
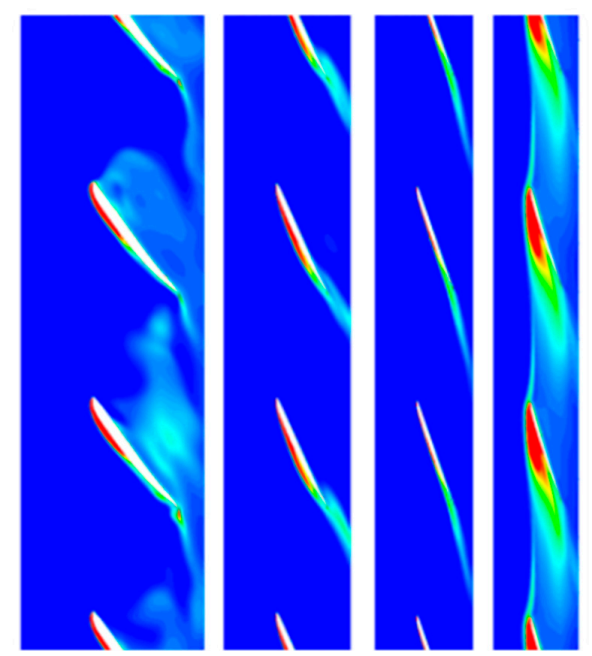

SP0.2

SP0.5 (b)
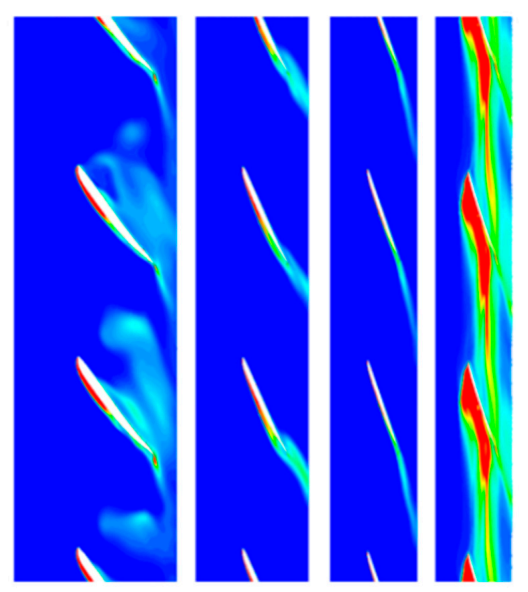

$\begin{array}{lll}\text { SP0.5 } & \text { SP0.8 } & \text { SP0.98 }\end{array}$

(d)

Figure 16. Distributions of $S_{D M}$ (reflecting the turbulent entropy generation rate) along different spanwise surfaces of the impeller for varying tip clearance at $\varphi=0.354((\mathbf{a}) \mathrm{h}=0.1 \%$ span; $(\mathbf{b}) \mathrm{h}=0.3 \%$ span; (c) $h=0.6 \%$ span; and (d) $h=0.8 \%$ span).

\subsection{Tip Flow Patterns and Distributions of the Turbulent Entropy Generation Rate in Varying Tip Gaps}

We introduced parameters to analyze the tip flow patterns in varying tip gaps. Figure 17 displays the definition of computational parameters in the tip gap. Planes relative to the blade were defined as $\lambda=S C^{-1}$, where $S$ is a coordinate aligned with the blade chord and $C$ is the blade chord from the leading edge (LE) to the trailing edge (TE). We here selected a plane at $\lambda=0.4$ for the following analysis presented in Figure 17b.

Detailed data of tip flow patterns at $\lambda=0.4$ for tip clearances of $0.1 \%$ span, $0.3 \%$ span, $0.6 \%$ span, and $0.8 \%$ span at the same flow rate $(\varphi=0.354)$ are shown in Figure 18. A positive axial velocity represents the mainstream while a negative velocity represents tip leakage flow. The non-dimensional mean axial velocity and streamlines for the tip clearance of $0.1 \%$ span displayed in Figure 18a reveal that the axial velocity in the gap was mostly negative and its distribution was uniform, which means that the tip leakage flow almost filled the gap and hardly mixed with the mainstream. Additionally, there was almost no tip separation vortex at the tip for $\lambda=0.4$ owing to strong effects of the viscous force at 
boundary layers. Compared with streamlines of a tip clearance of $0.1 \%$ span, Figure $18 b, c, d$ shows part of the positive axial velocity at the tip for $\lambda=0.4$, indicating that the mainstream mixes with leakage flow and the formation of a tip separation vortex in the gap. This tip separation vortex attaches more on the blade tip surface as the tip clearance increases from $0.3 \%$ span to $0.8 \%$ span, as seen in Figure $18 \mathrm{~b}-\mathrm{d}$. This is because the mainstream mixes more with the tip leakage flow to create more vortices for larger tip clearance at the same flow rate. As the tip clearance grows from $0.3 \%$ span to $0.8 \%$ span, the core of the tip leakage vortex moves closer to the blade suction side. The high turbulence of the tip separation vortex can connect with the shear layer and merge with the tip leakage vortex to block the tip flow and mainstream, finally increasing entropy generation. The tip flow patterns for tip clearances of $0.3 \%$ span, $0.6 \%$ span, and $0.8 \%$ span were similar to those modeled by Denton [28] (see Figure 31a in his paper). For the smallest tip clearance of $0.1 \%$ span, the tip flow structure was different from that in Denton's work because the tip flow hardly mixed with the mainstream at the tip. Denton's leakage mixing model assumes that the mainstream and leakage flow must mix at the tip, and his model is thus not suitable for a very small tip clearance of $0.1 \%$ span.

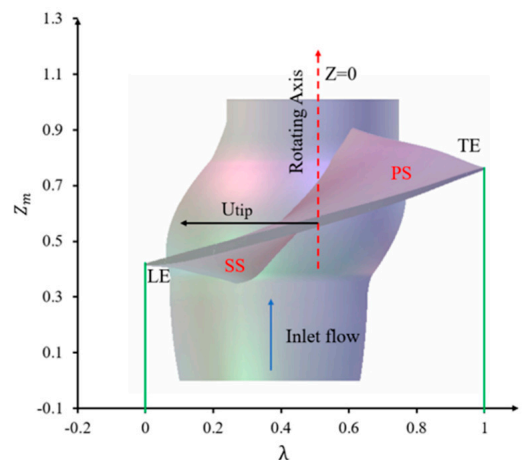

(a)

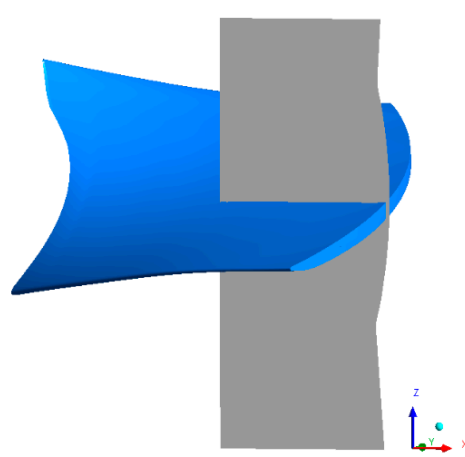

(b)

Figure 17. Parameter definition of the blade tip: (a) impeller's blade; and (b) selected plane at $\lambda=0.4$.
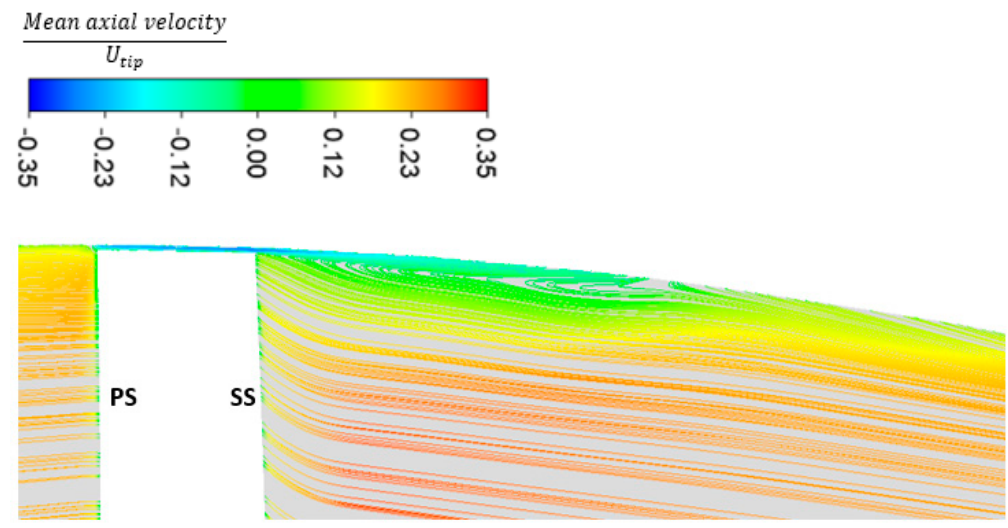

(a)

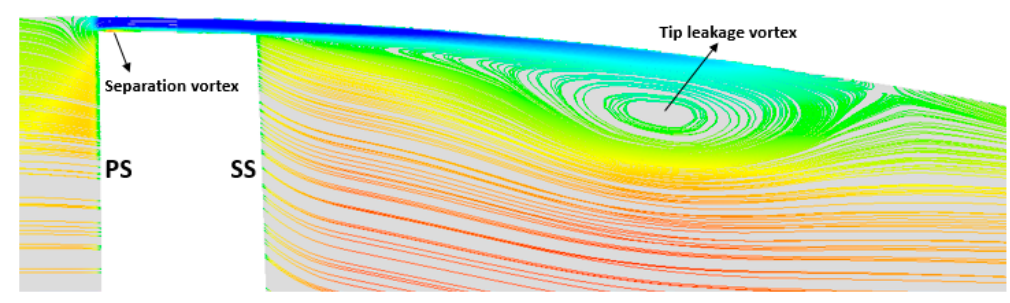

(b)

Figure 18. Cont. 


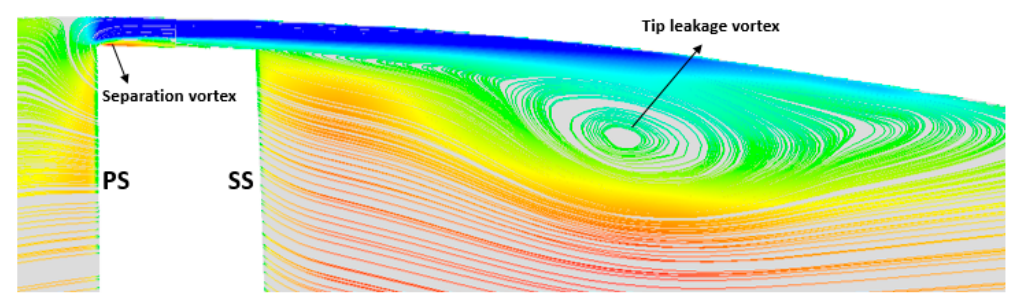

(c)

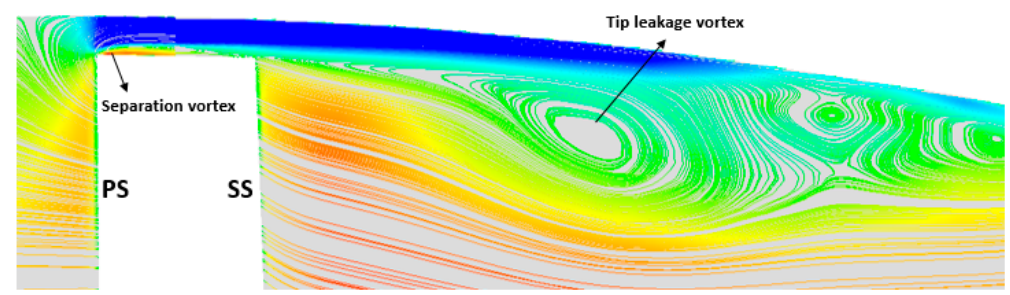

(d)

Figure 18. Distribution of non-dimensional mean axial velocities and streamlines at $\lambda=0.4$ for varying tip clearance at $\varphi=0.354((\mathbf{a}) \mathrm{h}=0.1 \%$ span; $(\mathbf{b}) \mathrm{h}=0.3 \% \mathrm{span} ;(\mathbf{c}) \mathrm{h}=0.6 \% \mathrm{span}$; and $(\mathbf{d}) \mathrm{h}=0.8 \% \mathrm{span})$.

To study distributions of the turbulent entropy generation rate for a varying tip gap, we selected the middle surface colored green along the blade chord direction at the tip for analysis as shown in Figure 19. We here used $S_{D M}$ (reflecting the turbulent entropy generation rate) on the selected plane in analyzing the distributions of turbulent dissipation in varying tip gaps at the same flow rate.

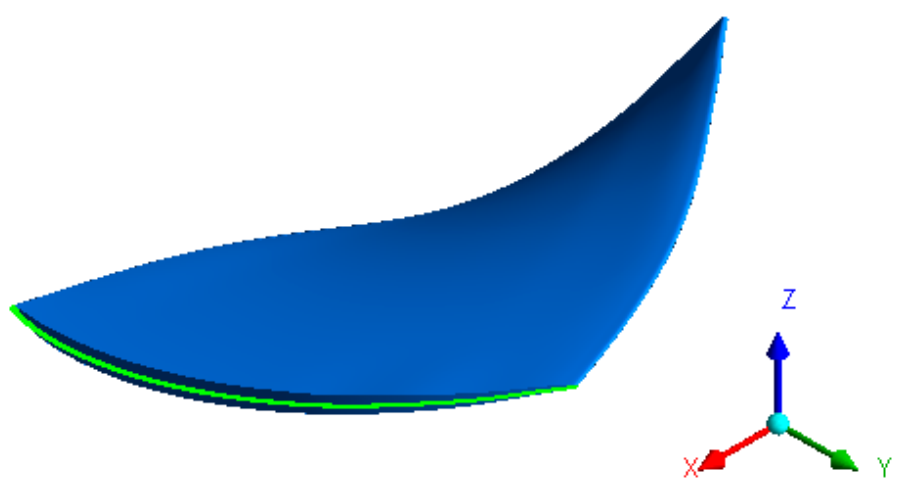

Figure 19. View of the selected plane along the blade chord direction at the tip.

Figure 20 displays distributions of $S_{D M}$ (reflecting the turbulent entropy generation rate) on the selected plane with a varying tip gap from the meridian view at the same flow rate $(\varphi=0.354)$. In the case of the tip clearance of $0.1 \%$ span presented in Figure 20a, the distribution of the turbulent entropy generation rate on the selected plane was uniform. For other tip clearances of $0.3 \%$ span, $0.6 \%$ span, and $0.8 \%$ span presented in Figure $20 \mathrm{~b}, \mathrm{c}, \mathrm{d}$, the turbulent entropy generation rate decreased from the blade leading edge (LE) to the trailing edge (TE). The large values of turbulent dissipation located near the leading edge of the blade tip side, originating from the leading edge and extending in the gap. The high entropy generation in these areas was due to the tip separation vortex attached to the blade tip side. Additionally, the area of high entropy generation on the selected plane in the tip increased with the tip clearance growing from $0.3 \%$ span to $0.8 \%$ span. This is because the area of the tip separation vortex occurred more and extended further for a larger tip clearance. In general, turbulent dissipation at the tip decreased from the blade leading edge to trailing edge, and the regions of high dissipation were distributed near the leading edge of the blade tip side. With the tip clearance increasing from 
$0.3 \%$ span to $0.8 \%$ span, the areas of high turbulent dissipation increase and extend further along the blade chord direction.

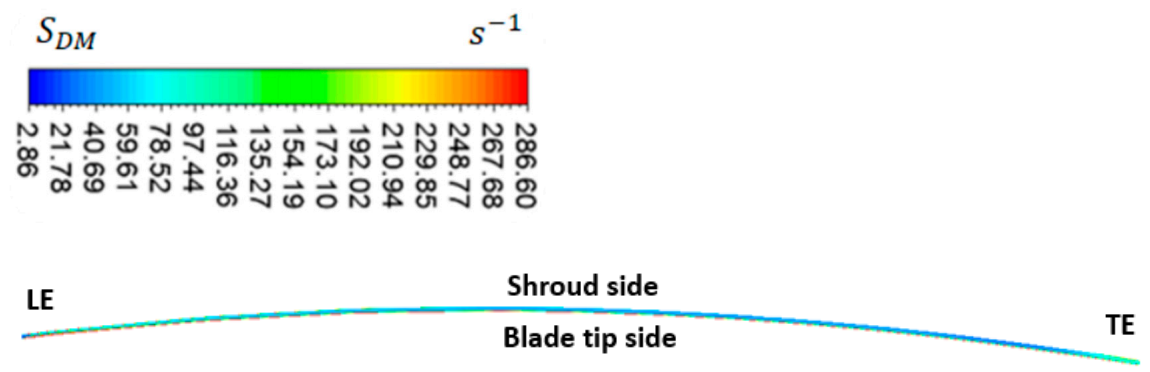

(a)

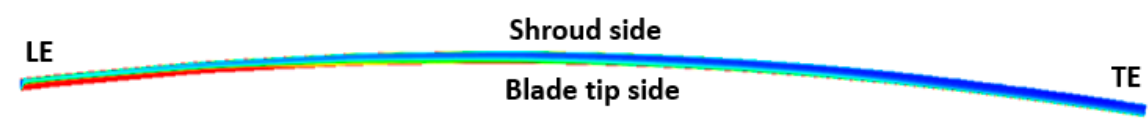

(b)

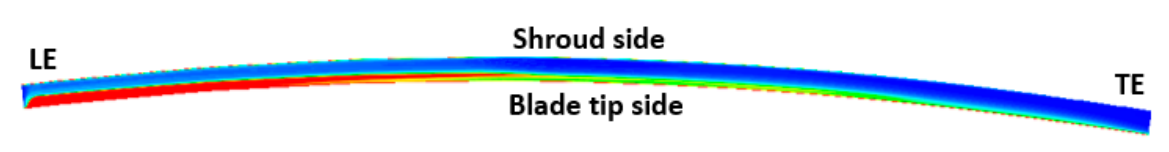

(c)

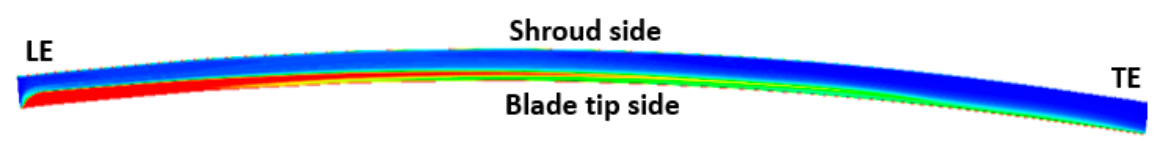

(d)

Figure 20. Distribution of $S_{D M}$ (reflecting the turbulent entropy generate rate) on the selected plane along the blade chord direction in a varying tip gap at $\varphi=0.354((\mathbf{a}) \mathrm{h}=0.1 \% \mathrm{span} ;(\mathbf{b}) \mathrm{h}=0.3 \% \mathrm{span}$; (c) $\mathrm{h}=0.6 \%$ span; and (d) $\mathrm{h}=0.8 \%$ span).

\section{Conclusions}

The effects of varying discharge and tip clearance on mechanical energy dissipation of an axial-flow pump were investigated using the entropy generation method in numerical simulations. The following conclusions were drawn by comparing the overall entropy generation rates for different components and analyzing the distributions of the turbulent entropy generation rate under varying conditions.

(1) The calculation results of an analysis of the overall entropy generation rate of different components of an axial-flow pump show that the mechanical energy dissipation in the impeller had a strong relation with the hump characteristic. Additionally, the impeller and diffuser were the main domains of high mechanical energy dissipation in the axial-flow pump under varying conditions, with the components respectively accounting for $35.32 \%-55.51 \%$ and $32.61 \%-20.42 \%$ of mechanical energy dissipation of the entire flow passage. Furthermore, the mechanical energy dissipation of the impeller, mostly being turbulent dissipation, became more important with the discharge decreasing. The discharge had greater effects on turbulent dissipation in the impeller when the axial-flow pump entered the hump region.

(2) When the pump entered the hump region, the distributions of turbulent dissipation near the shroud became disordered and expanded towards the impeller's inlet side. Unstable flows, like flow separation and vortices, near the blade suction sides led to the distribution of high turbulent dissipation in the impeller and the hump characteristic. Regions of high turbulent dissipation in the diffuser locate 
at the leading edges of guide vanes and in the diffuser passage. The distribution of high turbulent dissipation in the diffuser expanded to be more uniform as the discharge declined.

(3) A tip clearance larger than $0.3 \%$ span affected turbulent dissipation of the impeller more and finally affected the pump performance. When the tip clearance grew from $0.1 \%$ span to $0.8 \%$ span for the same discharge $(\varphi=0.354)$, areas of high turbulent dissipation grew obviously for span SP0.98 with the tip clearance increasing. Therefore, an increase in the tip clearance mainly increased turbulent dissipation near the shroud of the impeller.

(4) As for the tip clearance of $0.1 \%$ span, the mainstream hardly mixed with the leakage flow, and distributions of the axial velocity and turbulent entropy generation rate at $\lambda=0.4$ in the gap under the discharge ( $\varphi=0.354)$ were thus uniform. Denton's leakage mixing model assumes that the mainstream and leakage flow mix at the tip and is thus not suitable for a very small tip clearance of $0.1 \%$ span. For other tip clearances of $0.3 \%$ span, $0.6 \%$ span, and $0.8 \%$ span, the tip flow patterns in the gap were similar to the pattern in Figure 31a of Denton's paper [28]. With the tip clearance increasing from $0.3 \%$ span to $0.8 \%$ span, the area of the tip separation vortex at $\lambda=0.4$ attached more on the blade tip surface, and the core of the tip leakage vortex moved closer to the blade suction side. The turbulent dissipation at the tip decreased from the blade leading edge to trailing edge, and regions of high dissipation distributed near the leading edge of the blade tip side. The areas of high turbulent dissipation increased and extended further along the blade chord direction with an increasing tip clearance.

Author Contributions: Z.Q. designed the experiments and funded the research work; S.S. performed the experiments and conducted the simulations; S.S. analyzed the data and wrote the paper; B.J. reviewed the research results and edited the paper.

Acknowledgments: This research was funded by National Natural Science Foundation of China (Grant Nos.51779186) and Natural Science Foundation of Hubei Province (2017CFA048 and 2018CFA010).

Conflicts of Interest: The authors declare that they have no conflict of interest.

\section{Nomenclature}

\begin{tabular}{|c|c|c|c|}
\hline Q & mass flow rate $(\mathrm{kg} / \mathrm{s})$ & g & gravitational acceleration $\left(\mathrm{m} / \mathrm{s}^{2}\right)$ \\
\hline $\mathrm{Z}_{\mathrm{R}}$ & diameter of the impeller $(0.3 \mathrm{~m})$ & $\mathrm{H}$ & head (m) \\
\hline h & tip clearance width (\% span) & $\rho$ & water density $\left(\mathrm{kg} / \mathrm{m}^{3}\right)$ \\
\hline $\mathrm{T}$ & temperature $(\mathrm{K})$ & $\mathrm{k}(\mathrm{TKE})$ & turbulence kinetic energy \\
\hline$\varphi$ & flow coefficient : $\varphi=\frac{\mathrm{Q}}{\left(\frac{\Omega}{2 \pi}\right) \mathrm{D}^{3}}$ & $\psi$ & head coef ficient: $\psi=\frac{g H}{\left(\frac{\Omega}{2 \pi}\right)^{2} D^{2}}$ \\
\hline$\eta$ & pump efficiency $(\%): \eta=\frac{\mathrm{gQH}}{3600 \mathrm{M} \Omega}$ & $\vec{q}$ & heat flux density vector \\
\hline $\mathrm{u}, \mathrm{v}, \mathrm{w}$ & velocity component along $\mathrm{x}, \mathrm{y}, \mathrm{z}$ direction $(\mathrm{m} / \mathrm{s})$ & $\mathrm{s}$ & specific entropy \\
\hline$\Phi, \Phi_{\Theta}$ & entropy generation term & $\Omega$ & rotor angular velocity $(\mathrm{rad} / \mathrm{s})$ \\
\hline$U_{\text {tip }}$ & blade tip speed : $U_{\text {tip }}=\Omega * Z_{\mathrm{R}} / 2=22.80(\mathrm{~m} / \mathrm{s})$ & $T_{r e f}$ & inlet temperature (298K) \\
\hline$\overline{()}$ & mean component & ()$^{\prime}$ & fluctuating component \\
\hline$\dot{S}_{\mathrm{D}}$ & \multicolumn{3}{|l|}{ overall entropy generation rate for a domain $(\mathrm{W} / \mathrm{K})$} \\
\hline$\dot{\mathrm{S}}_{\overline{\mathrm{D}}}$ & \multicolumn{3}{|c|}{ overall entropy generation rate induced by time-averaged movement for a domain $(\mathrm{W} / \mathrm{K})$} \\
\hline$\dot{\mathrm{S}}_{\mathrm{D}^{\prime}}$ & \multicolumn{3}{|c|}{ overall entropy rate caused by velocity fluctuations for a domain(W/K) } \\
\hline$\dot{\mathrm{S}}_{\overline{\mathrm{D}}}^{\prime \prime}$ & \multicolumn{3}{|c|}{ entropy generation rate induced by time-averaged movement $\left(\mathrm{Wm}^{-3} \mathrm{~K}^{-1}\right)$} \\
\hline$\dot{S}_{D^{\prime}}^{\prime \prime}$ & \multicolumn{3}{|c|}{ entropy generation rate caused by velocity fluctuation $\left(\mathrm{Wm}^{-3} \mathrm{~K}^{-1}\right)$} \\
\hline
\end{tabular}

\section{References}

1. Kock, F.; Herwig, H. Entropy production calculation for turbulent shear flows and their implementation in CFD codes. Int. J. Heat Fluid Flow 2005, 26, 672-680. [CrossRef]

2. Herwig, H.; Kock, F. Direct and indirect methods of calculating entropy generation rates in turbulent convective heat transfer problems. Heat Mass Transf. 2007, 43, 207-215. [CrossRef] 
3. Herwig, H.; Kock, F. Local entropy production in turbulent shear flows: A tool for evaluating heat transfer performance. J. Therm. Sci. 2006, 15, 159-167. [CrossRef]

4. Gong, R.Z.; Wang, H.J.; Chen, L.X.; Li, D.Y.; Zhang, H.C.; Wei, X.Z. Application of entropy production theory to hydro-turbine hydraulic analysis. Sci. China Technol. Sci. 2013, 56, 1636-1643. [CrossRef]

5. Hou, H.; Zhang, Y.; Li, Z.; Jiang, T.; Zhang, J.; Xu, C. Numerical analysis of entropy production on a LNG cryogenic submerged pump. J. Nat. Gas Sci. Eng. 2016, 36, 87-96. [CrossRef]

6. Li, X.; Jiang, Z.; Zhu, Z.; Si, Q.; Li, Y. Entropy generation analysis for the cavitating head-drop characteristic of a centrifugal pump. Proc. Inst. Mech. Eng. Part C J. Mech. Eng. Sci. 2018, 232, 095440621775345. [CrossRef]

7. Pei, J.; Meng, F.; Li, Y.; Yuan, S.; Chen, J. Effects of distance between impeller and guide vane on losses in a low head pump by entropy production analysis. Adv. Mech. Eng. 2016, 8. [CrossRef]

8. Li, D.; Gong, R.; Wang, H.; Xiang, G.; Wei, X.; Qin, D. Entropy production analysis for hump characteristics of a pump turbine model. Chin. J. Mech. Eng. 2016, 29, 803-812. [CrossRef]

9. Li, D.; Wang, H.; Qin, Y.; Han, L.; Wei, X.; Qin, D. Entropy production analysis of hysteresis characteristic of a pump-turbine model. Energy Convers. Manag. 2017, 149, 175-191. [CrossRef]

10. Qian, Z.; Wang, Y.; Huai, W.; Lee, Y. Numerical simulation of water flow in an axial flow pump with adjustable guide vanes. J. Mech. Sci. Technol. 2010, 24, 971-976. [CrossRef]

11. Feng, J.; Luo, X.; Guo, P.; Wu, G. Influence of tip clearance on pressure fluctuations in an axial flow pump. J. Mech. Sci. Technol. 2016, 30, 1603-1610. [CrossRef]

12. Lei, S.; Desheng, Z.; Ruijie, Z.; Weidong, S.; Yongxin, J. Effect of blade tip geometry on tip leakage vortex dynamics and cavitation pattern in axial-flow pump. Sci. China Technol. Sci. 2017, 10, 46-59.

13. Zhang, D.; Shi, W.; Van Esch, B.P.M.; Shi, L.; Dubuisson, M. Numerical and experimental investigation of tip leakage vortex trajectory and dynamics in an axial flow pump. Comput. Fluids 2015, 112, 61-71. [CrossRef]

14. Long, Y.; Long, X.P.; Ji, B.; Huai, W.X.; Qian, Z.D. Verification and validation of URANS simulations of the turbulent cavitating flow around the hydrofoil. J. Hydrodyn. 2017, 29, 610-620. [CrossRef]

15. Cheng, H.Y.; Bai, X.R.; Long, X.P.; Ji, B.; Peng, X.X.; Farhat, M. Large Eddy Simulation of the Tip-leakage Cavitating flow with an insight on how cavitation influences vorticity and turbulence. Appl. Math. Model. 2020, 77, 788-809. [CrossRef]

16. Decaix, J.; Balarac, G.; Dreyer, M.; Farhat, M.; Münch, C. RANS and LES computations of the tip-leakage vortex for different gap widths. J. Turbul. 2015, 16, 309-341. [CrossRef]

17. Chang, H.; Shi, W.; Li, W.; Liu, J. Energy loss analysis of novel self-priming pump based on the entropy production theory. J. Therm. Sci. 2019, 28, 306-318. [CrossRef]

18. Wang, C.; Zhang, Y.; Hou, H.; Zhang, J.; Xu, C. Entropy production diagnostic analysis of energy consumption for cavitation flow in a two-stage LNG cryogenic submerged pump. Int. J. Heat Mass Transf. 2019, 129, 342-356. [CrossRef]

19. Li, D.; Wang, H.; Qin, Y.; Wei, X.; Qin, D. Numerical simulation of hysteresis characteristic in the hump region of a pump-turbine model. Renew. Energy 2018, 115, 433-447. [CrossRef]

20. Liu, Y.; Tan, L.; Hao, Y.; Xu, Y. Energy Performance and Flow Patterns of a Mixed-Flow Pump with Different Tip Clearance Sizes. Energies 2017, 10, 191. [CrossRef]

21. Li, S.; Zhang, A.M.; Han, R.; Ma, Q. 3D full coupling model for strong interaction between a pulsating bubble and a movable sphere. J. Comput. Phys. 2019, 392, 713-731. [CrossRef]

22. Kock, F.; Herwig, H. Local entropy production in turbulent shear flows: A high-reynolds number model with wall functions. Int. J. Heat Mass Transf. 2004, 47, 2205-2215. [CrossRef]

23. Mathieu, J.; Scott, J. An Introduction to Turbulent Flow; Cambridge University Press: Cambridge, UK, 2000.

24. Qian, Z.; Wang, F.; Guo, Z.; Lu, J. Performance evaluation of an axial-flow pump with adjustable guide vanes in turbine mode. Renew. Energy 2016, 99, 1146-1152. [CrossRef]

25. Shen, S.; Qian, Z.; Ji, B.; Agarwal, R.K. Numerical investigation of tip flow dynamics and main flow characteristics with varying tip clearance widths for an axial-flow pump. Proc. Inst. Mech. Eng. Part A J. Power Energy 2019, 233, 476-488. [CrossRef]

26. Feng, W.; Pan, J.; Guo, Z.; Cheng, Q. The effects of variable-inlet guide vanes on performance of an axial flow pump with tip clearance. Symposia 2015, 1, V001T33A017. 
27. Feng, W.; Cheng, Q.; Guo, Z.; Qian, Z. Simulation of cavitation performance of an axial flow pump with inlet guide vanes. Adv. Mech. Eng. 2016, 8, 1-8. [CrossRef]

28. Denton, J.D. Loss Mechanisms in Turbomachines. ASME J. Turbomach. 1993, 115, 621-656. [CrossRef]

(c)

(C) 2019 by the authors. Licensee MDPI, Basel, Switzerland. This article is an open access article distributed under the terms and conditions of the Creative Commons Attribution (CC BY) license (http://creativecommons.org/licenses/by/4.0/). 